\title{
Discrete Midpoint Convexity
}

\author{
Satoko Moriguchi;, Kazuo Murota, Akihisa Tamura; Fabio Tardella ${ }^{\S}$
}

August 2017; November 2018

\begin{abstract}
For a function defined on the integer lattice, we consider discrete versions of midpoint convexity, which offer a unifying framework for discrete convexity of functions, including integral convexity, $\mathrm{L}^{\natural}$-convexity, and submodularity. By considering discrete midpoint convexity for all pairs at $\ell_{\infty}$-distance equal to two or not smaller than two, we identify new classes of discrete convex functions, called locally and globally discrete midpoint convex functions. These functions enjoy nice structural properties. They are stable under scaling and addition, and satisfy a family of inequalities named parallelogram inequalities. Furthermore, they admit a proximity theorem with the same small proximity bound as that for $\mathrm{L}^{\natural}$-convex functions. These structural properties allow us to develop an algorithm for the minimization of locally and globally discrete midpoint convex functions based on the proximity-scaling approach and on a novel 2-neighborhood steepest descent algorithm.
\end{abstract}

Keywords: Midpoint convexity, Discrete convex function, Integral convexity, $\mathrm{L}^{\natural}$-convexity, Proximity theorem, Scaling algorithm.

\section{Introduction}

For a function $f$ defined on a convex set $S \subseteq \mathbb{R}^{n}$, midpoint convexity is the property requiring that

$$
f(x)+f(y) \geq 2 f\left(\frac{x+y}{2}\right)
$$

for all $x, y \in S$. It may be worthwhile to recall that the first definition and systematic study of convex functions appeared more than a century ago in a milestone paper by Jensen [21], where midpoint convexity was used to define convex functions. The now classical defining inequality for convex functions

$$
\alpha f(x)+(1-\alpha) f(y) \geq f(\alpha x+(1-\alpha) y) \quad(0 \leq \alpha \leq 1)
$$

\footnotetext{
${ }^{*}$ Department of Economics and Business Administration, Tokyo Metropolitan University, satoko5@tmu.ac.jp

'Department of Economics and Business Administration, Tokyo Metropolitan University, murota@tmu.ac.jp

${ }^{\ddagger}$ Department of Mathematics, Keio University, aki-tamura@math.keio.ac.jp

${ }^{\S}$ Department of Methods and Models for Economics, Territory and Finance, Sapienza University of Rome, fabio.tardella@uniroma1.it
} 
was then proved by Jensen to be implied by midpoint convexity in the case of continuous functions and of functions bounded above. In the same paper, the celebrated Jensen inequality was also derived from midpoint convexity. The equivalence between midpoint convexity (1.1) and classical convexity (1.2) was later shown to hold under very mild assumptions such as measurability or local boundedness above (see, e.g., [2], [39] and references therein). It is apparent that midpoint convexity implies nonnegativity of the second order directional differences $D_{h}^{2} f(x)=f(x+h)-2 f(x)+f(x-h)$ for all $x, h \in \mathbb{R}^{n}$ such that $x+h, x, x-h \in S$. In the case of twice continuously differentiable functions this clearly implies that the second order derivatives of $f$ are nonnegative in all directions $h$, and hence that the Hessian of $f$ is positive semidefinite.

For a function defined on the integer lattice $\mathbb{Z}^{n}$, we may consider analogous notions of discrete midpoint convexity where the value of the function at the (possibly nonintegral) midpoint is replaced by suitable convex combinations of the function values at some integer neighboring points.

For $x, y \in \mathbb{Z}^{n}$, one very simple substitute for $f((x+y) / 2)$ in the case of noninteger $(x+y) / 2$ is obtained by taking the average of the values of $f$ at the integer round-up and round-down of the point $(x+y) / 2$. In this case we obtain the inequality of discrete midpoint convexity:

$$
f(x)+f(y) \geq f\left(\left\lceil\frac{x+y}{2}\right\rceil\right)+f\left(\left\lfloor\frac{x+y}{2}\right\rfloor\right),
$$

where $\lceil\cdot\rceil$ and $\lfloor\cdot\rfloor$ denote the integer vectors obtained by rounding up and rounding down each component to the nearest integers, respectively.

A weaker version of discrete midpoint convexity is obtained by considering the smallest possible value of a linear extension of $f$ at the point $(x+y) / 2$ with respect to the values at the integer points neighboring $(x+y) / 2$. More precisely, for a point $x \in \mathbb{R}^{n}$ we consider its integer neighborhood $N(x)=\left\{z \in \mathbb{Z}^{n}|| x_{i}-z_{i} \mid<1(i=1, \ldots, n)\right\}$ and the value at $x$ of the convex envelope $\tilde{f}$ of $f$ on $N(x)$, which can be represented as

$$
\tilde{f}(x)=\min \left\{\sum_{z \in N(x)} \lambda_{z} f(z) \mid \sum_{z \in N(x)} \lambda_{z} z=x,\left(\lambda_{z}\right) \in \Lambda(x)\right\} \quad\left(x \in \mathbb{R}^{n}\right),
$$

where $\Lambda(x)$ denotes the set of coefficients $\left(\lambda_{z} \mid z \in N(x)\right) \in \mathbb{R}^{N(x)}$ for convex combinations indexed by $N(x)$. We then consider the following inequality of weak discrete midpoint convexity:

$$
f(x)+f(y) \geq 2 \tilde{f}\left(\frac{x+y}{2}\right)
$$

which is indeed a weaker condition than 1.3 since $\frac{x+y}{2}=\frac{1}{2}\left\lceil\frac{x+y}{2}\right\rceil+\frac{1}{2}\left\lfloor\frac{x+y}{2}\right\rfloor$ implies $2 \tilde{f}\left(\frac{x+y}{2}\right) \leq$ $f\left(\left\lceil\frac{x+y}{2}\right\rceil\right)+f\left(\left\lfloor\frac{x+y}{2}\right\rfloor\right)$ by 1.4 .

The objective of this paper is twofold: (i) to highlight discrete midpoint convexity as a unifying framework for convexity concepts of functions on the integer lattice $\mathbb{Z}^{n}$, and (ii) to investigate structural and algorithmic properties of functions defined by versions of discrete midpoint convexity.

New and known classes of functions can be obtained by requiring discrete midpoint convexity (1.3) or weak discrete midpoint convexity (1.5) for all points $x, y$ at a prescribed $\ell_{\infty^{-}}$ distance. It is known that weak discrete midpoint convexity (1.5) coincides with integral convexity [5], and that discrete midpoint convexity $\left[1.3\right.$ for all $x, y$ characterizes $L^{\natural}$-convexity [8, 30], whereas [1.3 for all $x, y$ at $\ell_{\infty}$-distance one characterizes submodularity [6] (see 
Section 2 for details). By requiring discrete midpoint convexity (1.3) for all pairs $(x, y)$ at $\ell_{\infty}$ distance equal to two or not smaller than two, we can define new classes of discrete convex functions, called locally and globally discrete midpoint convex functions, which are strictly between the classes of $\mathrm{L}^{\natural}$-convex and integrally convex functions.

Locally and globally discrete midpoint convex functions enjoy nice structural properties possessed by $\mathrm{L}^{\natural}$-convex functions and not by general integrally convex functions. Discrete midpoint convex functions are stable under addition and scaling (Proposition 2.13, Theorem 4.2), and satisfy a family of inequalities which are named parallelogram inequalities (Theorem 3.1). Furthermore, they admit a proximity theorem with the same small proximity bound as that for $\mathrm{L}^{\natural}$-convex functions (Theorem 5.1). These structural properties allow us to develop a proximity-scaling based algorithm for the minimization of locally and globally discrete midpoint convex functions.

Algorithms based on scaling and proximity have been successful for discrete optimization problems such as resource allocation problems [13, 14, 15, 22] and convex network flow problems (under the name of "capacity scaling") [1, 19, 20]. For separable convex functions defined on discrete rectangles (boxes), the proximity-scaling approach is straightforward. $\mathrm{L}^{\natural}$-convex functions are also amenable to this approach: the scaling operation preserves $\mathrm{L}^{\natural}$ convexity, and the proximity theorem holds. Efficient algorithms for minimizing $L^{\natural}$-convex functions have been successfully designed with the proximity-scaling approach [28, 30] and also for L-convex functions on graphs [10,11]. Recently a proximity-scaling algorithm has been developed for integrally convex functions in [27]. The algorithm proposed in this paper employs the standard proximity-scaling framework while using a novel variant of descent algorithm suited for discrete midpoint convex functions. Our descent algorithm, named the 2-neighborhood steepest descent algorithm, repeats finding a local minimizer in the neighborhood of $\ell_{\infty}$-distance 2 , and the number of iterations is shown to be exactly equal to the minimum $\ell_{\infty}$-distance to a minimizer, which extends the results of [23, 33] for $L^{\natural}$-convex functions. Our scaling algorithm finds a minimizer of a discrete midpoint convex function with $\mathrm{O}\left(5^{n} n \log _{2} K_{\infty}\right)$ function evaluations, where $K_{\infty}$ denotes the $\ell_{\infty}$-size of the effective domain of the function. This means that, if the dimension $n$ is fixed, the algorithm is polynomial in the problem size. Besides the proximity approach, there are many other approaches to nonlinear integer optimization [24]. Among others, methods based on sophisticated algebraic tools have been developed in the last two decades, as described in [4, 9, 36] as well as [24].

In the next section we describe in detail the relations of discrete midpoint convexity with integral convexity, $\mathrm{L}^{\natural}$-convexity, and submodularity. Furthermore, we introduce the new classes of locally and globally discrete midpoint convex sets and functions, and we analyze their basic properties. For locally and globally discrete midpoint convex functions we establish a useful "parallelogram inequality" in Section 3, while in Sections 4 and 5 we present scaling and proximity results. Such results are then used to develop a scaling algorithm for minimization in Section 6. Quadratic functions with discrete midpoint convexity are considered in Section 7, while Section 8 is devoted to technical proofs of basic facts about discrete midpoint convex sets.

\section{Discrete Midpoint Convexity}

In this section we first provide an overview of the relations between functions satisfying (weak) discrete midpoint convexity for all pairs of points at a prescribed $\ell_{\infty}$-distance and the classes of submodular, integrally convex, and $\mathrm{L}^{\natural}$-convex functions in the simpler case where 
the functions are defined on a finite rectangular domain (box) of $\mathbb{Z}^{n}$. Then we show that the same relations also hold in the case of more general domains. Furthermore, we show that there exist only two classes of discrete midpoint convex functions that do not coincide with previously known classes of functions. We call these new classes locally and globally discrete midpoint convex functions and we describe here their basic properties.

\subsection{New and known classes of discrete midpoint convex functions}

We introduce some notations to better illustrate various classes of discrete midpoint convex functions and their relations with other known classes of functions. We denote by $\mathrm{DMC}(k)$ and by $\mathrm{DMC}(\geq k)$ the classes of functions $f: \mathbb{Z}^{n} \rightarrow \mathbb{R} \cup\{+\infty\}$ that satisfy discrete midpoint convexity $(1.3)$ for all $x, y \in \mathbb{Z}^{n}$ with $\|x-y\|_{\infty}=k$ and for all $x, y \in \mathbb{Z}^{n}$ with $\|x-y\|_{\infty} \geq k$, respectively. Similarly, we denote by $\operatorname{WDMC}(k)$ and by $\operatorname{WDMC}(\geq k)$ the classes of functions that satisfy weak discrete midpoint convexity (1.5) for all $x, y \in \mathbb{Z}^{n}$ with $\|x-y\|_{\infty}=k$ and for all $x, y \in \mathbb{Z}^{n}$ with $\|x-y\|_{\infty} \geq k$, respectively.

We recall that a function $f: \mathbb{Z}^{n} \rightarrow \mathbb{R} \cup\{+\infty\}$ is submodular if

$$
f(x)+f(y) \geq f(x \vee y)+f(x \wedge y)
$$

for all $x, y \in \mathbb{Z}^{n}$, where $x \vee y$ and $x \wedge y$ denote the componentwise maximum and minimum of the vectors $x$ and $y$, respectively. As is well known, a function $f$ defined on a rectangular domain is submodular if and only if it satisfies the inequality (2.1) for every pair $(x, y)$ with $\|x-y\|_{\infty}=1$. Furthermore, since $x \vee y=\lceil(x+y) / 2\rceil$ and $x \wedge y=\lfloor(x+y) / 2\rfloor$ when $\|x-y\|_{\infty}=1$, the inequality (2.1) is equivalent to discrete midpoint convexity (1.3) for points at $\ell_{\infty}$-distance 1. Thus, on a rectangular domain, the class of submodular functions coincides with DMC(1).

In discrete convex analysis [29, 30, 31, 32], a variety of discrete convex functions are considered. A function $f: \mathbb{Z}^{n} \rightarrow \mathbb{R} \cup\{+\infty\}$ is called $\mathrm{L}^{\natural}$-convex if it satisfies translationsubmodularity:

$$
f(x)+f(y) \geq f((x-\mu \mathbf{1}) \vee y)+f(x \wedge(y+\mu \mathbf{1}))
$$

for all $x, y \in \mathbb{Z}^{n}$ and nonnegative integers $\mu$, where $\mathbf{1}=(1,1, \ldots, 1)$. It is known that $f$ is $\mathrm{L}^{\natural}$-convex if and only if it satisfies discrete midpoint convexity 1.3 for all $x, y \in \mathbb{Z}^{n}$, or, equivalently, for all points $x, y$ at $\ell_{\infty}$-distance 1 or 2 (see Theorem 2.8 ). Thus, the class of $\mathrm{L}^{\natural}$ convex functions coincides with the two equivalent classes $\mathrm{DMC}(\geq 1)=\mathrm{DMC}(1) \cap \mathrm{DMC}(2)$. $\mathrm{L}^{\natural}$-convex functions form a major class of discrete convex functions and have applications in several fields including image processing [23], auction theory [25, 34], inventory theory [38, 41], and scheduling [3].

Separable convex functions are a special case of $\mathrm{L}^{\mathrm{b}}$-convex functions. A function $f$ : $\mathbb{Z}^{n} \rightarrow \mathbb{R} \cup\{+\infty\}$ in $x=\left(x_{1}, \ldots, x_{n}\right)$ is called separable convex if it can be represented as $f(x)=\varphi_{1}\left(x_{1}\right)+\cdots+\varphi_{n}\left(x_{n}\right)$ with univariate discrete convex functions $\varphi_{i}: \mathbb{Z} \rightarrow \mathbb{R} \cup\{+\infty\}$ satisfying discrete midpoint convexity at distance two: $\varphi_{i}(t-1)+\varphi_{i}(t+1) \geq 2 \varphi_{i}(t)$ for all $t \in \mathbb{Z}$.

A function $f: \mathbb{Z}^{n} \rightarrow \mathbb{R} \cup\{+\infty\}$ is called integrally convex [5] if its local convex envelope $\tilde{f}: \mathbb{R}^{n} \rightarrow \mathbb{R} \cup\{+\infty\}$ defined by 1.4 is (globally) convex in the ordinary sense. Note that $\tilde{f}$ can be viewed as the collection of convex envelopes of $f$ in each unit hypercube $\left\{x \in \mathbb{R}^{n} \mid a_{i} \leq x_{i} \leq a_{i}+1(i=1, \ldots, n)\right\}$ with $a \in \mathbb{Z}^{n}$. Integrally convex functions constitute a common framework for discrete convex functions, including separable convex and $\mathrm{L}^{\natural}$-convex functions as well as $\mathrm{M}^{\natural}$-convex, $\mathrm{L}_{2}^{\natural}$-convex and $\mathrm{M}_{2}^{\natural}$-convex functions [30], and BS-convex and UJ-convex functions [7]. Submodular integrally convex functions are exactly $\mathrm{L}^{\natural}$-convex 


\begin{tabular}{|ll|}
\hline WDMC $(1)$ & $:$ all functions \\
$\operatorname{DMC}(1)$ & $:$ submodular \\
WDMC $(2)=$ WDMC $(\geq 1)$ & $:$ integrally convex \\
$\operatorname{DMC}(1) \cap \operatorname{WDMC}(2)$ & $:$ submodular integrally convex \\
$=\operatorname{DMC}(1) \cap \operatorname{DMC}(2)$ & \\
$=\mathrm{DMC}(\geq 1)$ & $: \mathrm{L}^{\natural}$-convex \\
$\varsubsetneqq \mathrm{DMC}(2) \cap \mathrm{DMC}(3)=\mathrm{DMC}(\geq 2)$ & $:$ globally discrete midpoint convex \\
$\varsubsetneqq \mathrm{DMC}(2)$ & $:$ locally discrete midpoint convex \\
$\varsubsetneqq \mathrm{WDMC}(2)$ & $:$ integrally convex \\
\hline
\end{tabular}

Figure 1: Function classes defined by discrete midpoint convexity (dom $f$ : discrete rectangle)

functions [8]. The concept of integral convexity is used in formulating discrete fixed point theorems [16, 17, 40]. In game theory the integral concavity of payoff functions guarantees the existence of a pure strategy equilibrium in finite symmetric games [18].

In the case of a rectangular domain, integrally convex functions were shown to coincide with the equivalent classes $\operatorname{WDMC}(2)=\operatorname{WDMC}(\geq 2)$ already in the seminal paper [5] (see Theorem 2.1). In the following section we clarify that the same relation holds in the case of integrally convex domains (to be defined below). For more general domains we might have $\operatorname{WDMC}(2) \neq \operatorname{WDMC}(\geq 2)$. However, as we show in Appendix A, WDMC $(\geq 2)$ always coincides with the class of integrally convex functions. To complete the picture, note that weak discrete midpoint convexity (1.5) trivially holds for any function and for all points $x, y$ at $\ell_{\infty}$-distance 1 . Thus WDMC(1) contains all functions.

After describing the classes $\operatorname{DMC}(1), \operatorname{DMC}(\geq 1)=\operatorname{DMC}(1) \cap \operatorname{DMC}(2), \operatorname{WDMC}(1)$, and $\operatorname{WDMC}(\geq 1)=\operatorname{WDMC}(1) \cap \operatorname{WDMC}(2)$, one might think of the classes $\operatorname{DMC}(k)$ or $\operatorname{DMC}(\geq k)$ or $\operatorname{WDMC}(k)$ or $\operatorname{WDMC}(\geq k)$ for $k \geq 3$. However, it does not seem interesting to consider such classes of functions that satisfy (weak) discrete midpoint convexity only for all $x, y \in \mathbb{Z}^{n}$ with $\|x-y\|_{\infty} \geq 3$, since this condition is not even sufficient for the standard one-dimensional discrete convexity on $\mathbb{Z}$. For example, the function $g: \mathbb{Z} \rightarrow \mathbb{R}$ with $g(0)=2$ and $g(z)=z^{2}$ for $z \neq 0$ satisfies discrete midpoint convexity for all $x, y \in \mathbb{Z}$ with $\|x-y\|_{\infty} \geq 3$, but is not discrete convex on $\mathbb{Z}$.

Thus, the only potentially interesting classes of discrete midpoint convex functions that have not yet been investigated are the classes $\operatorname{DMC}(2)$ and $\operatorname{DMC}(\geq 2)$, which we call locally discrete midpoint convex functions ${ }^{1}$ and globally discrete midpoint convex functions, respectively. We analyze their properties in Sections 2.4 and $3-5$, where we also prove that $\operatorname{DMC}(\geq 2)=\operatorname{DMC}(2) \cap \operatorname{DMC}(3)$ (Proposition 3.3), thus showing that no intermediate class of discrete midpoint convex functions exists between locally and globally midpoint convex functions.

\subsection{Weak discrete midpoint convexity and integral convexity}

Recall that a function $f: \mathbb{Z}^{n} \rightarrow \mathbb{R} \cup\{+\infty\}$ is said to be integrally convex [5] if its local convex envelope $\tilde{f}: \mathbb{R}^{n} \rightarrow \mathbb{R} \cup\{+\infty\}$ defined by $(1.4)$ with respect to the integral neighborhood $N(x)=\left\{z \in \mathbb{Z}^{n}|| x_{i}-z_{i} \mid<1(i=1, \ldots, n)\right\}\left(x \in \mathbb{R}^{n}\right)$ is convex on $\mathbb{R}^{n}$.

\footnotetext{
${ }^{1}$ Locally discrete midpoint convex functions have been previously called directed integrally convex functions in the preliminary paper [26] which anticipates part of the results of this paper.
} 
A set $S \subseteq \mathbb{Z}^{n}$ is said to be integrally convex if the convex hull $\bar{S}$ of $S$ coincides with the union of the convex hulls of $S \cap N(x)$ over $x \in \mathbb{R}^{n}$, i.e., if, for any $x \in \mathbb{R}^{n}, x \in \bar{S}$ implies $x \in \overline{S \cap N(x)}$. A set $S \subseteq \mathbb{Z}^{n}$ is integrally convex if and only if its indicator function $\delta_{S}$ is an integrally convex function, where the indicator function $\delta_{S}$ is defined by $\delta_{S}(x)=0$ for $x \in S$ and $\delta_{S}(x)=+\infty$ for $x \notin S$. An integrally convex set is "hole-free" in the sense of $S=\bar{S} \cap \mathbb{Z}^{n}$. The effective domain $\operatorname{dom} f=\left\{x \in \mathbb{Z}^{n} \mid f(x)<+\infty\right\}$ and the set of minimizers of an integrally convex function $f$ are both integrally convex [30, Proposition 3.28].

Integral convexity can be characterized by a local condition under the assumption that the effective domain is an integrally convex set. The following theorem is proved in [5, Proposition 3.3] when the effective domain is an integer interval (discrete rectangle) and in the general case in [27, Theorem 2.3].

Theorem 2.1 ([5, 27]). Let $f: \mathbb{Z}^{n} \rightarrow \mathbb{R} \cup\{+\infty\}$ be a function with an integrally convex effective domain. Then the following properties are equivalent:

(a) $f$ is integrally convex.

(b) For every $x, y \in \mathbb{Z}^{n}$ with $\|x-y\|_{\infty}=2$ we have

$$
f(x)+f(y) \geq 2 \tilde{f}\left(\frac{x+y}{2}\right) .
$$

Since integral convexity clearly implies weak discrete midpoint convexity for any pair of points $x, y \in \mathbb{Z}^{n}$, and the condition in part (b) of Theorem 2.1 is the local version of weak discrete midpoint convexity that defines $\operatorname{WDMC}(2)$, we obtain the following characterization of integral convexity in terms of "local" or "global" weak discrete midpoint convexity.

Corollary 2.2. Let $f: \mathbb{Z}^{n} \rightarrow \mathbb{R} \cup\{+\infty\}$ be a function with an integrally convex effective domain. Then the following properties are equivalent.

(a) $f$ is integrally convex.

(b) $f \in \operatorname{WDMC}(2)$.

(c) $f \in \operatorname{WDMC}(\geq 1)$.

One of the most useful properties of convex functions is the local characterization of global minima. This property holds for integrally convex functions in the following form.

Theorem 2.3 ([5, Proposition 3.1]; see also [30, Theorem 3.21]). Let $f: \mathbb{Z}^{n} \rightarrow \mathbb{R} \cup\{+\infty\}$ be an integrally convex function and $x^{*} \in \operatorname{dom} f$. Then $x^{*}$ is a minimizer of $f$ if and only if $f\left(x^{*}\right) \leq f\left(x^{*}+d\right)$ for all $d \in\{-1,0,+1\}^{n}$.

Theorem 2.3 above can be generalized to the following "box-barrier property" (see Fig. 2), of which Theorem 2.3 is a special case with $p=\hat{x}-\mathbf{1}$ and $q=\hat{x}+\mathbf{1}$.

Theorem 2.4 (Box-barrier property [26, 27]). Let $f: \mathbb{Z}^{n} \rightarrow \mathbb{R} \cup\{+\infty\}$ be an integrally convex function, and let $p \in(\mathbb{Z} \cup\{-\infty\})^{n}$ and $q \in(\mathbb{Z} \cup\{+\infty\})^{n}$, where $p \leq q$. Define

$$
\begin{aligned}
S & =\left\{x \in \mathbb{Z}^{n} \mid p_{i}<x_{i}<q_{i}(i=1, \ldots, n)\right\}, \\
W_{i}^{+} & =\left\{x \in \mathbb{Z}^{n} \mid x_{i}=q_{i}, p_{j} \leq x_{j} \leq q_{j}(j \neq i)\right\} \quad(i=1, \ldots, n), \\
W_{i}^{-} & =\left\{x \in \mathbb{Z}^{n} \mid x_{i}=p_{i}, p_{j} \leq x_{j} \leq q_{j}(j \neq i)\right\} \quad(i=1, \ldots, n),
\end{aligned}
$$

and $W=\bigcup_{i=1}^{n}\left(W_{i}^{+} \cup W_{i}^{-}\right)$. Let $\hat{x} \in S \cap \operatorname{dom} f$. If $f(\hat{x}) \leq f(y)$ for all $y \in W$, then $f(\hat{x}) \leq f(z)$ for all $z \in \mathbb{Z}^{n} \backslash S$.

\footnotetext{
${ }^{2}$ (a) and (c) are equivalent without the assumption of $\operatorname{dom} f$ being integrally convex; see Appendix A
} 


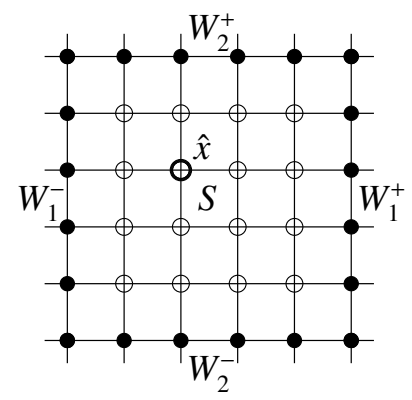

Figure 2: Box-barrier property $(\circ \in S, \bullet \in W)$

\subsection{Discrete midpoint convex sets}

We call a set $S \subseteq \mathbb{Z}^{n}$ discrete midpoint convex if

$$
x, y \in S,\|x-y\|_{\infty} \geq 2 \Longrightarrow\left\lceil\frac{x+y}{2}\right\rceil,\left\lfloor\frac{x+y}{2}\right\rfloor \in S .
$$

For comparison we recall [30] that a set $S \subseteq \mathbb{Z}^{n}$ is called $\mathrm{L}^{\natural}$-convex if $\lceil(x+y) / 2\rceil,\lfloor(x+y) / 2\rfloor \in$ $S$ for all $x, y \in S$.

\section{Proposition 2.5.}

(1) An $\mathrm{L}^{\natural}$-convex set is discrete midpoint convex.

(2) A discrete midpoint convex set is integrally convex.

Proof. (1) is obvious, whereas the proof of (2) is given in Section 8.1

The following proposition states some straightforward properties of discrete midpoint convex sets.

\section{Proposition 2.6.}

(1) Every subset of $\{0,1\}^{n}$ is discrete midpoint convex.

(2) The intersection of two (or more) discrete midpoint convex sets is discrete midpoint convex.

Remark 2.1. The inclusion relations among the classes of $\mathrm{L}^{\natural}$-convex sets, discrete midpoint convex sets, and integrally convex sets are all proper. That is, $\left\{\mathrm{L}^{\natural}\right.$-convex sets $\} \varsubsetneqq$ \{discrete midpoint convex sets $\} \varsubsetneqq$ integrally convex sets $\}$. For example, the set $\{(1,0),(0,1)\}$ is a discrete midpoint convex set that is not $\mathrm{L}^{\natural}$-convex. The set $\left\{\left(x_{1}, x_{2}\right) \in \mathbb{Z}^{2} \mid x_{1}+x_{2}=0\right\}$ is an integrally convex set that is not discrete midpoint convex.

Remark 2.2. A set $S \subseteq \mathbb{Z}^{n}$ is called $L_{2}^{\natural}$-convex if it can be represented as the Minkowski sum of two $\mathrm{L}^{\natural}$-convex sets. There is no inclusion relation between $\mathrm{L}_{2}^{\natural}$-convex sets and discrete midpoint convex sets. For example, the set $\{(1,0),(0,1)\}$ is a discrete midpoint convex set that is not $\mathrm{L}_{2}^{\natural}$-convex. The set $\{(0,0,0,0),(0,1,1,0),(1,1,0,0),(1,2,1,0)\}$ is an $\mathrm{L}_{2}^{\natural}$-convex set that is not discrete midpoint convex. Indeed, this set is the Minkowski sum of two $\mathrm{L}^{\natural}$-convex sets $\{(0,0,0,0),(1,1,0,0)\}$ and $\{(0,0,0,0),(0,1,1,0)\}$, and 2.4$)$ fails for $x=(0,0,0,0)$ and $y=(1,2,1,0)$. 


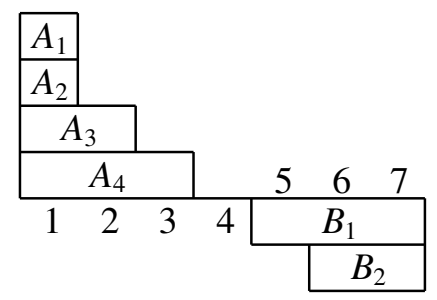

Figure 3: Subsets $A_{k}$ and $B_{k}$ in 2.6) for $y-x=(4,2,1,0,-1,-2,-2)$ in Example 2.1

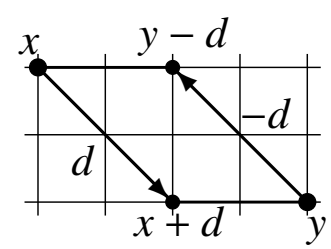

Figure 4: Parallelogram property

Besides midpoint convexity, a convex set $S$ in $\mathbb{R}^{n}$ has the property that for two points $x$ and $y$ in $S$ and a direction vector $d=t(y-x)$ from $x$ to $y$ with $0 \leq t \leq 1$, both $x+d$ and $y-d$ belong to $S$. Theorem 2.7 below shows a discrete analogue of this property, where the direction vector $d$ is discretized in a specific manner.

For any pair of distinct vectors $x, y \in \mathbb{Z}^{n}$, we can consider a decomposition of $y-x$ into vectors of $\{-1,0,+1\}^{n}$ as

$$
y-x=\sum_{k=1}^{m}\left(\mathbf{1}_{A_{k}}-\mathbf{1}_{B_{k}}\right),
$$

where $m=\|y-x\|_{\infty}, \mathbf{1}_{A}$ is the characteristic vector of $A \subseteq\{1, \ldots, n\}$,

$$
A_{k}=\left\{i \mid y_{i}-x_{i} \geq m+1-k\right\}, \quad B_{k}=\left\{i \mid y_{i}-x_{i} \leq-k\right\} \quad(k=1, \ldots, m) .
$$

Note that $A_{1} \subseteq A_{2} \subseteq \cdots \subseteq A_{m}, B_{1} \supseteq B_{2} \supseteq \cdots \supseteq B_{m}, A_{m} \cap B_{1}=\emptyset$, and $A_{1} \cup B_{m} \neq \emptyset$.

Example 2.1. Figure 3 shows the subsets $A_{k}$ and $B_{k}$ in 2.6 for $x=(0,0,0,0,0,0,0)$ and $y=(4,2,1,0,-1,-2,-2)$. We have $m=4,\left(A_{1}, A_{2}, A_{3}, A_{4}\right)=(\{1\},\{1\},\{1,2\},\{1,2,3\})$ and $\left(B_{1}, B_{2}, B_{3}, B_{4}\right)=(\{5,6,7\},\{6,7\}, \emptyset, \emptyset)$. Accordingly, the decomposition $(2.5)$ is given by $y-$ $x=(4,2,1,0,-1,-2,-2)=(1,0,0,0,-1,-1,-1)+(1,0,0,0,0,-1,-1)+(1,1,0,0,0,0,0)+$ $(1,1,1,0,0,0,0)$.

Theorem 2.7. Let $S \subseteq \mathbb{Z}^{n}$ be a discrete midpoint convex set, $x, y \in S$, and $d=\sum_{k \in J}\left(\mathbf{1}_{A_{k}}-\mathbf{1}_{B_{k}}\right)$ for some $J \subseteq\{1,2, \ldots, m\}$. Then $x+d \in S$ and $y-d \in S$.

Proof. The proof is given in Section 8.2 .

The property shown in Theorem 2.7 will be referred to as the "parallelogram property" of discrete midpoint convex sets, as a parallelogram is formed by the four points $x, x+d, y$, and $y-d$ (cf. Fig. 4). This property will be used to establish the "parallelogram inequality," a key property of discrete midpoint convex functions, in Theorem 3.1 . 


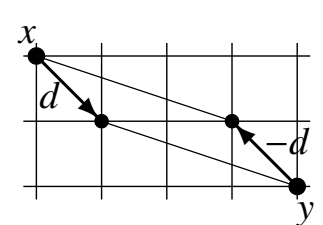

$J=\{1\}, d=(1,-1)$

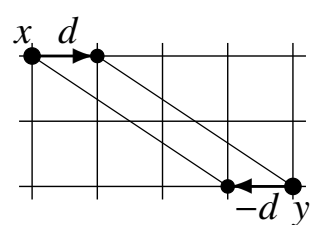

$J=\{3\}, d=(1,0)$

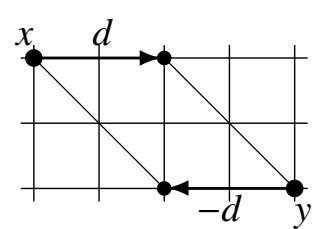

$J=\{3,4\}, d=(2,0)$

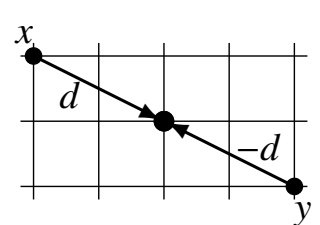

$J=\{1,3\}, d=(2,-1)$

Figure 5: Parallelogram property for $x=(0,0), y=(4,-2)$ in Example 2.2

Example 2.2. For $x=(0,0)$ and $y=(4,-2)$, the decomposition $(2.5)$ is given as $y-x=$ $(4,-2)=(1,-1)+(1,-1)+(1,0)+(1,0)$ with $m=4,\left(A_{1}, B_{1}\right)=\left(A_{2}, B_{2}\right)=(\{1\},\{2\})$, and $\left(A_{3}, B_{3}\right)=\left(A_{4}, B_{4}\right)=(\{1\}, \emptyset)$. Suppose that $x$ and $y$ are contained in a discrete midpoint convex set $S$. Different choices of $J \subseteq\{1,2,3,4\}$ in Theorem 2.7 generate different points $x+d, y-d$ contained in $S$, as illustrated in Fig. 5 .

\subsection{Discrete midpoint convex functions}

In this section we consider discrete midpoint convexity

$$
f(x)+f(y) \geq f\left(\left\lceil\frac{x+y}{2}\right\rceil\right)+f\left(\left\lfloor\frac{x+y}{2}\right\rfloor\right)
$$

for functions $f: \mathbb{Z}^{n} \rightarrow \mathbb{R} \cup\{+\infty\}$.

In Section 2.1 we have defined $\mathrm{L}^{\natural}$-convex functions in terms of translation-submodularity (2.2). However, $\mathrm{L}^{\natural}$-convexity can be viewed as a type of discrete midpoint convexity by the following known result.

Theorem $2.8\left([5,8,[30])\right.$. For $f: \mathbb{Z}^{n} \rightarrow \mathbb{R} \cup\{+\infty\}$ the following conditions, (a) to (d), are equivalent: $:^{3}$

(a) $f$ satisfies translation-submodularity (2.2).

(b) $f$ satisfies discrete midpoint convexity (2.7) for all $x, y \in \mathbb{Z}^{n}$, i.e., $f \in \mathrm{DMC}(\geq 1)$.

(c) $f$ satisfies discrete midpoint convexity (2.7) for all $x, y \in \mathbb{Z}^{n}$ with $\|x-y\|_{\infty} \leq 2$, i.e., $f \in \mathrm{DMC}(1) \cap \mathrm{DMC}(2)$, and the effective domain has the property: $x, y \in \operatorname{dom} f \Rightarrow$ $\lceil(x+y) / 2\rceil,\lfloor(x+y) / 2\rfloor \in \operatorname{dom} f$.

(d) $f$ is integrally convex and submodular

We call a function $f: \mathbb{Z}^{n} \rightarrow \mathbb{R} \cup\{+\infty\}$ locally discrete midpoint convex if $\operatorname{dom} f$ is a discrete midpoint convex set and the discrete midpoint convexity (2.7) is satisfied by every pair $(x, y) \in \mathbb{Z}^{n} \times \mathbb{Z}^{n}$ with $\|x-y\|_{\infty}=2$ (exactly equal to 2 ). We call a function $f: \mathbb{Z}^{n} \rightarrow$ $\mathbb{R} \cup\{+\infty\}$ globally discrete midpoint convex if the discrete midpoint convexity (2.7) is satisfied by every pair $(x, y) \in \mathbb{Z}^{n} \times \mathbb{Z}^{n}$ with $\|x-y\|_{\infty} \geq 2$. The effective domain of a globally discrete midpoint convex function is necessarily a discrete midpoint convex set.

Proposition 2.9. For a globally discrete midpoint convex function $f$, the effective domain $\operatorname{dom} f$ is a discrete midpoint convex set.

\footnotetext{
${ }^{3} \mathbb{Z}$-valued functions are treated in [8, Theorem 3], but the proof is valid for $\mathbb{R}$-valued functions.

${ }^{4}$ If $\operatorname{dom} f$ is a discrete rectangular domain (box), condition (d) can be written " $f \in \operatorname{DMC}(1) \cap \operatorname{WDMC}(2)$ " as in Fig. 1 .
} 
Proof. It follows from (2.7) for $\|x-y\|_{\infty} \geq 2$ that $S=\operatorname{dom} f$ satisfies (2.4).

We have the following inclusion relations among the function classes:

$$
\begin{aligned}
& \left\{L^{\natural} \text {-convex }\right\}=\{\text { submodular integrally convex }\} \\
& \varsubsetneqq\{\text { globally discrete midpoint convex }\} \\
& \varsubsetneqq\{\text { locally discrete midpoint convex }\} \\
& \varsubsetneqq\{\text { integrally convex }\},
\end{aligned}
$$

as proved below. Using the notation of the Introduction, we can express $(2.8)$ as $\mathrm{DMC}(\geq 1) \varsubsetneqq$ $\operatorname{DMC}(\geq 2) \varsubsetneqq \operatorname{DMC}(2) \varsubsetneqq \operatorname{WDMC}(\geq 1)$.

\section{Theorem 2.10.}

(1) An $\mathrm{L}^{\natural}$-convex function is globally discrete midpoint convex.

(2) A globally discrete midpoint convex function is locally discrete midpoint convex.

(3) A locally discrete midpoint convex function is integrally convex.

Proof. (1) This is immediate from the characterizations of $\mathrm{L}^{\natural}$-convex functions by discrete midpoint convexity in Theorem 2.8 .

(2) Let $f$ be a globally discrete midpoint convex function. By Proposition 2.9, $\operatorname{dom} f$ is a discrete midpoint convex set. The discrete midpoint convexity (2.7) when $\|x-y\|_{\infty}=2$ is obviously true, since it is true when $\|x-y\|_{\infty} \geq 2$.

(3) Let $f$ be a locally discrete midpoint convex function. Then $\operatorname{dom} f$ is a discrete midpoint convex set, which is an integrally convex set by Proposition 2.5. We use the characterization of integrally convex functions stated in Theorem 2.1. Recall that, for $u \in \mathbb{R}^{n}, \tilde{f}(u)$ denotes the local convex extension of $f$ with respect to the integer neighborhood $N(u)$. Take integer points $x, y \in \operatorname{dom} f$ with $\|x-y\|_{\infty}=2$. For $u=(x+y) / 2$, both $\lceil u\rceil$ and $\lfloor u\rfloor$ belong to $N(u)$, and therefore $2 \tilde{f}(u) \leq f(\lceil u\rceil)+f(\lfloor u\rfloor) \leq f(x)+f(y)$, where the second inequality is due to (2.7). Thus, $f$ satisfies condition (b) in Theorem 2.1, and therefore it is integrally convex.

The inclusions in (2.8) are all proper. The examples in Remark 2.1 in Section 2.3 show this for the first and third " $\varsubsetneqq$ " since a set $S \subseteq \mathbb{Z}^{n}$ is discrete midpoint convex if and only if its indicator function $\delta_{S}$ is a (globally or locally) discrete midpoint convex function. The difference between global and local versions is demonstrated in the following examples.

Example 2.3. $f\left(x_{1}, x_{2}\right)=\left|x_{1}+x_{2}\right|$ is a locally discrete midpoint convex function on $\operatorname{dom} f=$ $\mathbb{Z}^{2}$, satisfying the discrete midpoint convexity 2.7) when $\|x-y\|_{\infty}=2$. For $x=(0,0)$ and $y=(3,-3)$ with $\|x-y\|_{\infty}=3$ we have $\lceil(x+y) / 2\rceil=(2,-1)$ and $\lfloor(x+y) / 2\rfloor=(1,-2)$, and therefore, $f(x)+f(y)=0+0, f\left(\left\lceil\frac{x+y}{2}\right]\right)+f\left(\left\lfloor\frac{x+y}{2}\right\rfloor\right)=1+1$. This shows a failure of 2.7. Hence $f$ is not globally discrete midpoint convex.

Example 2.4. Let $f(x)=x^{\top} Q x$ with $Q=\left[\begin{array}{ll}1 & c \\ c & 1\end{array}\right]$ for $x \in \mathbb{Z}^{2}$. It can be verified from the definitions (or by Propositions 7.3, 7.6, and 7.5 in Section 7) that

- $f$ is $\mathrm{L}^{\natural}$-convex if and only if $-1 \leq c \leq 0$,

- $f$ is globally discrete midpoint convex if and only if $-1 \leq c \leq 4 / 5$,

- $f$ is locally discrete midpoint convex if and only if $-1 \leq c \leq 1$. 
Further relations among the function classes in some special cases are shown next.

Proposition 2.11.

(1) Every function on the unit hypercube $\{0,1\}^{n}$ is globally (and hence locally) discrete midpoint convex.

(2) For $n=2$, every integrally convex function on a discrete midpoint convex set is locally discrete midpoint convex ${ }^{5}$

(3) Let $f$ be a submodular function on $\mathbb{Z}^{n}$. Then $f$ is integrally convex if and only if it is (globally or locally) discrete midpoint convex.

Proof. (1) There exist no $x, y \in\{0,1\}^{n}$ with $\|x-y\|_{\infty}=2$. Therefore, the condition for global discrete midpoint convexity is satisfied vacuously.

(2) Suppose that $f$ is integrally convex, and take any $x, y \in \mathbb{Z}^{2}$ with $\|x-y\|_{\infty}=2$, and let $u=(x+y) / 2$. Since $n=2, N(u)=\{\lceil u\rceil,\lfloor u\rfloor\}$ if $u \notin \mathbb{Z}^{2}$ and $N(u)=\{u\}$ if $u \in \mathbb{Z}^{2}$. Hence, the integral convexity (2.3) in Theorem 2.1 implies the discrete midpoint convexity (2.7).

(3) By Theorem 2.10 or (2.8), a (globally or locally) discrete midpoint convex function is integrally convex, irrespective of submodularity. If $f$ is a submodular function that is integrally convex, then it is $\mathrm{L}^{\natural}$-convex by Theorem 2.8 , and hence globally discrete midpoint convex by Theorem 2.10 (1). This completes the proof of (3), since global discrete midpoint convexity implies local discrete midpoint convexity by Theorem 2.10(2).

In the case of $n=2$ the inclusion relations in (2.8) are modified to

$$
\begin{aligned}
& \left\{\mathrm{L}^{\natural} \text {-convex }\right\} \varsubsetneqq\{\text { globally discrete midpoint convex }\} \\
& \varsubsetneqq\{\text { locally discrete midpoint convex }\}=\{\text { integrally convex }\}
\end{aligned}
$$

by Example 2.4 and Proposition 2.11(2).

For discrete midpoint convexity of the minimizers we have the following statements.

Proposition 2.12. For a globally discrete midpoint convex function $f$, the set of the minimizers $\arg \min f$ is a discrete midpoint convex set.

Proof. It follows from (2.7) that $S=\arg \min f$ satisfies (2.4).

Remark 2.3. The set of the minimizers of a locally discrete midpoint convex function $f$ is not necessarily a discrete midpoint convex set. For example, $f\left(x_{1}, x_{2}\right)=\left|x_{1}+x_{2}\right|$ is locally discrete midpoint convex on $\mathbb{Z}^{2}$, but $\arg \min f=\{(t,-t) \mid t \in \mathbb{Z}\}$ is not a discrete midpoint convex set. It should be clear that no local version of discrete midpoint convexity is defined for sets.

Simple operations valid for globally or locally discrete midpoint convex functions are listed below. The scaling operation will be treated separately in Section 4.

Proposition 2.13. Let $f: \mathbb{Z}^{n} \rightarrow \mathbb{R} \cup\{+\infty\}$ be globally discrete midpoint convex functions.

(1) For any $z \in \mathbb{Z}^{n}, f(z+x)$ is globally discrete midpoint convex in $x$.

(2) For any permutation $\sigma$ of $(1,2, \ldots, n), f\left(x_{\sigma(1)}, x_{\sigma(2)}, \ldots, x_{\sigma(n)}\right)$ is globally discrete midpoint convex in $x$.

(3) $f\left(-x_{1},-x_{2}, \ldots,-x_{n}\right)$ is globally discrete midpoint convex in $x$.

(4) For any $a_{1}, a_{2} \geq 0$ and globally discrete midpoint convex functions $f_{1}, f_{2}: \mathbb{Z}^{n} \rightarrow \mathbb{R} \cup\{+\infty\}$, function $g=a_{1} f_{1}+a_{2} f_{2}$ is globally discrete midpoint convex. That is, the class of globally discrete midpoint convex functions forms a convex cone.

\footnotetext{
${ }^{5}$ Example 2.3 shows that we cannot replace "locally" with "globally" in this statement.
} 
Proof. (1)-(3) Obvious. (4) The inequality (2.7) for $g$ follows immediately from adding (2.7) for $a_{1} f_{1}$ and $a_{2} f_{2}$.

Proposition 2.14. The statements (1)-(4) in Proposition 2.13 are true for locally discrete midpoint convex functions.

Proof. (1)-(3) Obvious. (4) The effective domain dom $g$ is discrete midpoint convex, since $\operatorname{dom} g=\operatorname{dom} f_{1} \cap \operatorname{dom} f_{2}$ and the intersection of two discrete midpoint convex sets is discrete midpoint convex (Proposition 2.6 (2)). The rest of the proof is the same as that of Proposition 2.13 (4).

Remark 2.4. For a (globally or locally) discrete midpoint convex function $f$, the function with individual sign inversion of variables, $f\left(s_{1} x_{1}, s_{2} x_{2}, \ldots, s_{n} x_{n}\right)$ with $s_{i} \in\{+1,-1\}(i=$ $1,2, \ldots, n)$, is not necessarily (globally or locally) discrete midpoint convex in $x$, though it remains integrally convex in $x$. For example, $f\left(x_{1}, x_{2}, x_{3}\right)=\max \left(x_{1}, x_{2}, x_{3}\right)$ is globally discrete midpoint convex, but $g\left(x_{1}, x_{2}, x_{3}\right)=f\left(x_{1}, x_{2},-x_{3}\right)=\max \left(x_{1}, x_{2},-x_{3}\right)$ is not even locally discrete midpoint convex, since it fails to satisfy the inequality (2.7) for $x=(0,0,0)$ and $y=(-2,-1,1)$. Indeed, we have $\lceil(x+y) / 2\rceil=(-1,0,1)$ and, $\lfloor(x+y) / 2\rfloor=(-1,-1,0)$, and therefore, $g(x)+g(y)=0+(-1), g\left(\left\lceil\frac{x+y}{2}\right\rceil\right)+g\left(\left\lfloor\frac{x+y}{2}\right\rfloor\right)=0+0$.

Remark 2.5. The concept of L-extendable functions [10] is defined for functions on the product of trees in terms of some variant of discrete midpoint convexity. When specialized to functions on $\mathbb{Z}^{n}$, a function $f: \mathbb{Z}^{n} \rightarrow \mathbb{R} \cup\{+\infty\}$ is L-extendable if and only if $f(x)=g(2 x)$ for some UJ-convex [7] function $g: \mathbb{Z}^{n} \rightarrow \mathbb{R} \cup\{+\infty\}$. The class of L-extendable functions does not contain, nor is contained by, the class of (globally or locally) discrete midpoint convex functions, as demonstrated by Examples 2.5 and 2.6 below. In the case of $n=2$, however, the class of L-extendable functions coincides with that of locally discrete midpoint convex functions (see also (2.9)).

Example 2.5. The function $f: \mathbb{Z}^{3} \rightarrow \mathbb{R} \cup\{+\infty\}$ defined by $f(1,0,0)=f(0,1,0)=f(0,0,1)=$ 0 with $\operatorname{dom} f=\{(1,0,0),(0,1,0),(0,0,1)\}$ is not L-extendable by [12, Theorem 1], whereas it is globally discrete midpoint convex by Proposition 2.11(1).

Example 2.6. The function $f: \mathbb{Z}^{3} \rightarrow \mathbb{R}$ defined by $f\left(x_{1}, x_{2}, x_{3}\right)=\max \left(x_{1}, x_{2},-x_{3}\right)$ is Lextendable, whereas it is not locally discrete midpoint convex. The L-extendability follows from the facts that (i) $g\left(x_{1}, x_{2}, x_{3}\right)=\max \left(x_{1}, x_{2}, x_{3}\right)$ is $\mathrm{L}^{\natural}$-convex, (ii) every $\mathrm{L}^{\natural}$-convex function is L-extendable, and (iii) if a function is L-extendable, so is the function with individual sign inversion of variables. However, the discrete midpoint convexity (2.7) fails for $f$ with $x=(0,0,0)$ and $y=(-2,-1,1)$; see Remark 2.4.

Remark 2.6. For a univariate discrete convex function $]^{6} \varphi: \mathbb{Z} \rightarrow \mathbb{R} \cup\{+\infty\}$, we call diffconvex and sum-convex the functions of two variables of the form $\varphi(s-t)$ and $\varphi(s+t)$, respectively, where $(s, t) \in \mathbb{Z}^{2}$. It is straightforward to verify that diff-convex and sum-convex functions are locally discrete midpoint convex. A function $f: \mathbb{Z}^{n} \rightarrow \mathbb{R} \cup\{+\infty\}$ is called 2-separable convex [10] if it can be expressed as the sum of univariate convex, diff-convex and sum-convex functions, i.e., if

$$
f\left(x_{1}, \ldots, x_{n}\right)=\sum_{i=1}^{n} \varphi_{i}\left(x_{i}\right)+\sum_{i \neq j} \varphi_{i j}\left(x_{i}-x_{j}\right)+\sum_{i \neq j} \psi_{i j}\left(x_{i}+x_{j}\right),
$$

\footnotetext{
${ }^{6}$ A univariate discrete convex function means a function $\varphi: \mathbb{Z} \rightarrow \mathbb{R} \cup\{+\infty\}$ such that $\varphi(t-1)+\varphi(t+1) \geq 2 \varphi(t)$ for all $t \in \mathbb{Z}$.
} 
where $\varphi_{i}, \varphi_{i j}, \psi_{i j}: \mathbb{Z} \rightarrow \mathbb{R} \cup\{+\infty\}(i, j=1, \ldots, n ; i \neq j)$ are univariate convex functions. A function $f$ is called 2-separable diff-convex if the sum-convex terms are not involved in (2.10), i.e., if

$$
f\left(x_{1}, \ldots, x_{n}\right)=\sum_{i=1}^{n} \varphi_{i}\left(x_{i}\right)+\sum_{i \neq j} \varphi_{i j}\left(x_{i}-x_{j}\right) .
$$

It is known that a 2-separable diff-convex function is $L^{\natural}$-convex, [30, 31] and a 2-separable convex function is L-extendable [10]. However, a 2-separable convex function is not necessarily locally discrete midpoint convex. For example, consider $f\left(x_{1}, x_{2}, x_{3}\right)=\left(x_{1}+x_{2}\right)^{2}$ for $n=3$. This is a 2-separable convex function that is not locally discrete midpoint convex. Indeed, for $x=(1,0,0)$ and $y=(0,1,2)$, we have $z=\left\lceil\frac{x+y}{2}\right\rceil=(1,1,1), w=\left\lfloor\frac{x+y}{2}\right\rfloor=(0,0,1)$, and $f(x)+f(y)=1+1<f(z)+f(w)=4+0$.

\section{Parallelogram Inequality}

An interesting inequality, to be named "parallelogram inequality," can be established for (globally or locally) discrete midpoint convexity functions.

Theorem 3.1 (Parallelogram inequality). Let $f: \mathbb{Z}^{n} \rightarrow \mathbb{R} \cup\{+\infty\}$ be a (globally or locally) discrete midpoint convex function, $x \in \operatorname{dom} f$, and $\left\{A_{1}, A_{2}, \ldots, A_{m} ; B_{1}, B_{2}, \ldots, B_{m}\right\}$ be a multiset of subsets of $\{1,2, \ldots, n\}$ such that $A_{1} \subseteq A_{2} \subseteq \cdots \subseteq A_{m}, B_{1} \supseteq B_{2} \supseteq \cdots \supseteq B_{m}, A_{m} \cap B_{1}=\emptyset$, $A_{1} \cup B_{m} \neq \emptyset$. For any bipartition $(I, J)$ of $\{1,2, \ldots, m\}(I \cap J=\emptyset$ and $I \cup J=\{1,2, \ldots, m\})$ we have

$$
f(x)+f\left(x+d_{1}+d_{2}\right) \geq f\left(x+d_{1}\right)+f\left(x+d_{2}\right),
$$

where $d_{1}=\sum_{i \in I}\left(\mathbf{1}_{A_{i}}-\mathbf{1}_{B_{i}}\right)$ and $d_{2}=\sum_{j \in J}\left(\mathbf{1}_{A_{j}}-\mathbf{1}_{B_{j}}\right)$.

Proof. For $y=x+d_{1}+d_{2}=x+\sum_{k=1}^{m}\left(\mathbf{1}_{A_{k}}-\mathbf{1}_{B_{k}}\right)$ we may assume $y \in \operatorname{dom} f$, since otherwise 3.1 is trivially true with $f\left(x+d_{1}+d_{2}\right)=+\infty$. Let $d_{1}^{1}, \ldots, d_{1}^{|I|}$ denote the vectors $\mathbf{1}_{A_{i}}-\mathbf{1}_{B_{i}}$ $(i \in I)$, and similarly, $d_{2}^{1}, \ldots, d_{2}^{|J|}$ the vectors $\mathbf{1}_{A_{j}}-\mathbf{1}_{B_{j}}(j \in J)$. For $k=0,1, \ldots,|I|$ and $l=0,1, \ldots,|J|$ define

$$
x(k, l)=x+\sum_{i=1}^{k} d_{1}^{i}+\sum_{j=1}^{l} d_{2}^{j} .
$$

By Theorem 2.7, we have $x(k, l) \in \operatorname{dom} f$ for all $(k, l)$ with $0 \leq k \leq|I|$ and $0 \leq l \leq|J|$. Note that (3.1) is rewritten as

$$
f(x(0,0))+f(x(|I|,|J|)) \geq f(x(|I|, 0))+f(x(0,|J|)) .
$$

Let $1 \leq k \leq|I|$ and $1 \leq l \leq|J|$. We make use of the following properties of the vectors $d_{1}^{k}$ and $d_{2}^{l}$ :

$$
\left\|d_{1}^{k}+d_{2}^{l}\right\|_{\infty}=2, \quad\left\lceil\left(d_{1}^{k}+d_{2}^{l}\right) / 2\right\rceil=\max \left(d_{1}^{k}, d_{2}^{l}\right), \quad\left\lfloor\left(d_{1}^{k}+d_{2}^{l}\right) / 2\right\rfloor=\min \left(d_{1}^{k}, d_{2}^{l}\right),
$$

where $\max (\cdot, \cdot)$ and $\min (\cdot, \cdot)$ denote the vectors of componentwise maximum and minimum, respectively. This follows from the fact that $d_{1}^{k}=\mathbf{1}_{A_{i}}-\mathbf{1}_{B_{i}}$ for some $i=i_{k} \in I$ and $d_{2}^{l}=\mathbf{1}_{A_{j}}-\mathbf{1}_{B_{j}}$ 
for some $j=j_{l} \in J$, where $\left(A_{i} \cap A_{j}\right) \cup\left(B_{i} \cap B_{j}\right)$ is nonempty and either $\left[A_{i} \subseteq A_{j}, B_{i} \supseteq B_{j}\right.$, $\left.A_{j} \cap B_{i}=\emptyset\right]$ or $\left[A_{j} \subseteq A_{i}, B_{j} \supseteq B_{i}, A_{i} \cap B_{j}=\emptyset\right]$ according as $i<j$ or $i>j$.

Since $\|x(k, l)-x(k-1, l-1)\|_{\infty}=\left\|d_{1}^{k}+d_{2}^{l}\right\|_{\infty}=2$, we can use the discrete midpoint convexity 2.7 to obtain

$$
\begin{aligned}
& f(x(k-1, l-1))+f(x(k, l)) \\
& \geq f(\lceil(x(k-1, l-1)+x(k, l)) / 2\rceil)+f(\lfloor(x(k-1, l-1)+x(k, l)) / 2\rfloor) .
\end{aligned}
$$

By $x(k, l)=x(k-1, l-1)+d_{1}^{k}+d_{2}^{l}$ we have

$$
\begin{aligned}
& \lceil(x(k-1, l-1)+x(k, l)) / 2\rceil \\
& =x(k-1, l-1)+\left\lceil\left(d_{1}^{k}+d_{2}^{l}\right) / 2\right\rceil \\
& =x(k-1, l-1)+\max \left(d_{1}^{k}, d_{2}^{l}\right) \\
& = \begin{cases}x(k-1, l-1)+d_{1}^{k}=x(k, l-1) & \left(i_{k}>j_{l}\right), \\
x(k-1, l-1)+d_{2}^{l}=x(k-1, l) & \left(i_{k}<j_{l}\right),\end{cases} \\
& \lfloor(x(k-1, l-1)+x(k, l)) / 2\rfloor \\
& =x(k-1, l-1)+\left\lfloor\left(d_{1}^{k}+d_{2}^{l}\right) / 2\right\rfloor \\
& =x(k-1, l-1)+\min \left(d_{1}^{k}, d_{2}^{l}\right) \\
& = \begin{cases}x(k-1, l-1)+d_{2}^{l}=x(k-1, l) & \left(i_{k}>j_{l}\right), \\
x(k-1, l-1)+d_{1}^{k}=x(k, l-1) & \left(i_{k}<j_{l}\right) .\end{cases}
\end{aligned}
$$

This shows that the right-hand side of $(3.3)$ is equal to $f(x(k, l-1))+f(x(k-1, l))$. Therefore,

$$
f(x(k-1, l-1))+f(x(k, l)) \geq f(x(k, l-1))+f(x(k-1, l)) .
$$

By adding these inequalities for $(k, l)$ with $1 \leq k \leq|I|$ and $1 \leq l \leq|J|$, we obtain (3.2). It is emphasized that all the terms that are cancelled in this addition of inequalities are finitevalued, since $x(k, l) \in \operatorname{dom} f$ for all $(k, l)$ with $0 \leq k \leq|I|$ and $0 \leq l \leq|J|$, by Theorem 2.7.

Theorem 3.1 can be recast into the following form, which is more convenient in some applications. See also Figs. 4 and 5 .

Theorem 3.2. Let $f: \mathbb{Z}^{n} \rightarrow \mathbb{R} \cup\{+\infty\}$ be a (globally or locally) discrete midpoint convex function, $x, y \in \operatorname{dom} f$, and $d=\sum_{k \in J}\left(\mathbf{1}_{A_{k}}-\mathbf{1}_{B_{k}}\right)$ for some $J \subseteq\{1,2, \ldots, m\}$ in $(2.5)$. Then

$$
f(x)+f(y) \geq f(x+d)+f(y-d) .
$$

As an application of the parallelogram inequality we show some properties of locally discrete midpoint convex functions. By definition, they satisfy inequality (2.7) for $x$ and $y$ with $\|x-y\|_{\infty}=2$. Here we are interested in this inequality for distant pairs $(x, y)$ with $\|x-y\|_{\infty} \geq 3$. Example 2.3 shows that 2.7) is not always satisfied if $\|x-y\|_{\infty} \geq 3$.

Proposition 3.3. Let $f: \mathbb{Z}^{n} \rightarrow \mathbb{R} \cup\{+\infty\}$ be a locally discrete midpoint convex function.

(1) Discrete midpoint convexity (2.7) holds for all $x, y \in \mathbb{Z}^{n}$ with $x \leq y$.

(2) Discrete midpoint convexity (2.7) holds for all $x, y \in \mathbb{Z}^{n}$ with $\|x-y\|_{\infty}$ even.

(3) If, in addition, (2.7) holds for all $x, y \in \mathbb{Z}^{n}$ with $\|x-y\|_{\infty}=3$, then (2.7) holds for all $x, y \in \mathbb{Z}^{n}$ with $\|x-y\|_{\infty} \geq 2$, that is, $f$ is globally discrete midpoint convex. 
Proof. Consider the representation $y-x=\sum_{k=1}^{m}\left(\mathbf{1}_{A_{k}}-\mathbf{1}_{B_{k}}\right)$ as in 2.5$)$, where $m=\|x-y\|_{\infty}$, $A_{1} \subseteq A_{2} \subseteq \cdots \subseteq A_{m}, B_{1} \supseteq B_{2} \supseteq \cdots \supseteq B_{m}, A_{m} \cap B_{1}=\emptyset$, and $A_{1} \cup B_{m} \neq \emptyset$.

(1) We use the parallelogram inequality (3.1) in Theorem 3.1 . For

$$
I=\left\{\begin{array}{ll}
\{m, m-2, \ldots, 2\} & (m: \text { even }), \\
\{m, m-2, \ldots, 1\} & (m: \text { odd }),
\end{array} \quad J= \begin{cases}\{m-1, m-3, \ldots, 1\} & (m: \text { even }), \\
\{m-1, m-3, \ldots, 2\} & (m: \text { odd }),\end{cases}\right.
$$

we can verify

$$
\begin{aligned}
& d_{1}=\sum_{i \in I}\left(\mathbf{1}_{A_{i}}-\mathbf{1}_{B_{i}}\right)=\lceil(y-x) / 2\rceil=\lceil(x+y) / 2\rceil-x, \\
& d_{2}=\sum_{j \in J}\left(\mathbf{1}_{A_{j}}-\mathbf{1}_{B_{j}}\right)=\lfloor(y-x) / 2\rfloor=\lfloor(x+y) / 2\rfloor-x,
\end{aligned}
$$

where $B_{k}=\emptyset$ for all $k$ by $x \leq y$. Then the parallelogram inequality (3.1) yields the inequality (2.7).

(2) For $I=\{2,4, \ldots, m\}$ and $J=\{1,3, \ldots, m-1\}$, we have $d_{1}=\sum_{i \in I}\left(\mathbf{1}_{A_{i}}-\mathbf{1}_{B_{i}}\right)=$ $\lceil(y-x) / 2\rceil=\lceil(x+y) / 2\rceil-x$ and $d_{2}=\sum_{j \in J}\left(\mathbf{1}_{A_{j}}-\mathbf{1}_{B_{j}}\right)=\lfloor(y-x) / 2\rfloor=\lfloor(x+y) / 2\rfloor-x$. Then the parallelogram inequality (3.1) yields $(2.7)$.

(3) By induction on $m=2,3,4, \ldots$, we prove that 2.7 holds for all $x, y \in \mathbb{Z}^{n}$ with $\|x-y\|_{\infty}=m$. By assumption this is true for $m=2,3$. Take $x, y \in \mathbb{Z}^{n}$ with $\|x-y\|_{\infty}=m$, where $m \geq 4$. In the representation $y-x=\sum_{k=1}^{m}\left(\mathbf{1}_{A_{k}}-\mathbf{1}_{B_{k}}\right)$ we may assume $A_{1} \neq \emptyset$; otherwise interchange $x$ and $y$. By the parallelogram inequality in Theorem 3.2 with $d=\mathbf{1}_{A_{1}}-\mathbf{1}_{B_{1}}$ we have $f(x)+f(y) \geq f(x+d)+f(y-d)=f\left(x^{\prime}\right)+f\left(y^{\prime}\right)$, where $x^{\prime}=x+d$ and $y^{\prime}=y-d$. Since $2 \leq\left\|x^{\prime}-y^{\prime}\right\|_{\infty} \leq m-1$, the induction hypothesis gives

$$
f\left(x^{\prime}\right)+f\left(y^{\prime}\right) \geq f\left(\left[\frac{x^{\prime}+y^{\prime}}{2}\right\rceil\right)+f\left(\left\lfloor\frac{x^{\prime}+y^{\prime}}{2}\right\rfloor\right)=f\left(\left\lceil\frac{x+y}{2}\right\rceil\right)+f\left(\left\lfloor\frac{x+y}{2}\right\rfloor\right) .
$$

Hence (2.7) holds for $m$.

In the notation of the Introduction, Proposition $3.3(2)$ shows $\operatorname{DMC}(2)=\bigcap_{k=1}^{\infty} \operatorname{DMC}(2 k)$, and Proposition 3.3 (3) shows $\mathrm{DMC}(2) \cap \mathrm{DMC}(3)=\mathrm{DMC}(\geq 2)$.

Remark 3.1. The local characterization of global minima for discrete midpoint convex functions, which is given in Theorem 2.3 for integrally convex functions, can be derived easily from the parallelogram inequality in Theorem 3.1 .

\section{Scaling Operations}

In designing efficient algorithms for discrete or combinatorial optimization, the proximityscaling approach is a fundamental technique. For a function $f: \mathbb{Z}^{n} \rightarrow \mathbb{R} \cup\{+\infty\}$ in integer variables and a positive integer $\alpha$, called a scaling unit, the $\alpha$-scaling of $f$ means the function $f^{\alpha}$ defined by $f^{\alpha}(x)=f(\alpha x)\left(x \in \mathbb{Z}^{n}\right)$. The scaled function $f^{\alpha}$ is simpler and hence easier to minimize. The quality of the obtained minimizer of $f^{\alpha}$ as an approximation to the minimizer of $f$ is guaranteed by a proximity theorem, which states that a (local) minimum of the scaled function $f^{\alpha}$ is close to a minimizer of the original function $f$.

Discrete midpoint convexity is stable under the scaling operation, which is the case for sets and functions. We denote the set of positive integers by $\mathbb{Z}_{++}$and the zero vector by $\mathbf{0}$. 
Proposition 4.1. Let $S \subseteq \mathbb{Z}^{n}$ be a discrete midpoint convex set and $\alpha \in \mathbb{Z}_{++}$. Then the scaled set $S^{\alpha}=\left\{x \in \mathbb{Z}^{n} \mid \alpha x \in S\right\}$ is discrete midpoint convex.

Proof. Let $S \subseteq \mathbb{Z}^{n}$ be a discrete midpoint convex set. It suffices to show that, if $\mathbf{0}, \alpha x \in S$ and $\|x\|_{\infty} \geq 2$, then $\alpha\lfloor x / 2\rfloor, \alpha\lceil x / 2\rceil \in S$. Since $\mathbf{0}, \alpha x \in S$ and $S$ is integrally convex by Proposition 2.5 (2), we have $\{\mathbf{0}, x, 2 x, \ldots,(\alpha-1) x, \alpha x\} \subseteq \bar{S} \cap \mathbb{Z}^{n}=S$. The discrete midpoint convexity 2.4 for the pair $(k x,(k+1) x)$ of consecutive points implies

$$
k x+\lfloor x / 2\rfloor, k x+\lceil x / 2\rceil \in S \quad(k=0,1, \ldots, \alpha-1) .
$$

Note that $k x$ and $(k+1) x$ are at distance $\|k x-(k+1) x\|_{\infty}=\|x\|_{\infty} \geq 2$, which allows us to use (2.4). We will show

$$
\begin{array}{ll}
k x+(\beta+1)\lfloor x / 2\rfloor \in S & (k=0,1, \ldots, \alpha-1-\beta), \\
k x+(\beta+1)\lceil x / 2\rceil \in S & (k=0,1, \ldots, \alpha-1-\beta),
\end{array}
$$

respectively, by induction on $\beta=0,1, \ldots, \alpha-1$. For $\beta=\alpha-1$, we have $k=0$ from $0 \leq k \leq \alpha-1-\beta$, and (4.2) and (4.3) coincide, respectively, with $\alpha\lfloor x / 2\rfloor \in S$ and $\alpha\lceil x / 2\rceil \in S$. For $\beta=0,4.2$ and (4.3) are already shown in (4.1).

Assume now that (4.2) holds for some $\beta \geq 0$, and let $k \in\{0,1, \ldots, \alpha-2-\beta\}$. Consider consecutive points $k x+(\beta+1)\lfloor x / 2\rfloor$ and $(k+1) x+(\beta+1)\lfloor x / 2\rfloor$, and note that their $\ell_{\infty}$-distance is equal to $\|x\|_{\infty}$. By applying the discrete midpoint convexity (2.4) to this pair of consecutive points we obtain

$$
k x+(\beta+1)\lfloor x / 2\rfloor+\lfloor x / 2\rfloor=k x+(\beta+2)\lfloor x / 2\rfloor \in S .
$$

This shows (4.2) for $\beta+1$. By induction we can conclude that $(4.2)$ is true for all $\beta$.

The other claim 4.3 can be proved in a similar manner. For a pair of consecutive points $k x+(\beta+1)\lceil x / 2\rceil$ and $(k+1) x+(\beta+1)\lceil x / 2\rceil$, the discrete midpoint convexity (2.4) yields

$$
k x+(\beta+1)\lceil x / 2\rceil+\lceil x / 2\rceil=k x+(\beta+2)\lceil x / 2\rceil \in S .
$$

Then (4.3) follows by induction on $\beta$.

\section{Theorem 4.2.}

(1) Let $f: \mathbb{Z}^{n} \rightarrow \mathbb{R} \cup\{+\infty\}$ be a globally discrete midpoint convex function and $\alpha \in \mathbb{Z}_{++}$. Then the scaled function $f^{\alpha}$ is globally discrete midpoint convex.

(2) Let $f: \mathbb{Z}^{n} \rightarrow \mathbb{R} \cup\{+\infty\}$ be a locally discrete midpoint convex function and $\alpha \in \mathbb{Z}_{+_{+}}$. Then the scaled function $f^{\alpha}$ is locally discrete midpoint convex.

Proof. In either case, $\operatorname{dom} f$ is a discrete midpoint convex set; this is true by definition in the local case, and by Proposition 2.9 in the global case. By Proposition 4.1 , dom $f^{\alpha}$ is a discrete midpoint convex set. Then Proposition 4.3 below establishes (1) and (2).

\section{Proposition 4.3.}

(1) Let $f$ be a globally discrete midpoint convex function. If $\mathbf{0}, \alpha x \in \operatorname{dom} f$ and $\|x\|_{\infty} \geq 2$, then

$$
f(\mathbf{0})+f(\alpha x) \geq f(\alpha\lfloor x / 2\rfloor)+f(\alpha\lceil x / 2\rceil) .
$$

(2) Let $f$ be a locally discrete midpoint convex function. If $\mathbf{0}, \alpha x \in \operatorname{dom} f$ and $\|x\|_{\infty}=2$, then (4.4) holds. 
Proof. The following proof works for both (1) and (2). Since $\operatorname{dom} f$ is an integrally convex set by Proposition 2.5 (2) with Proposition 2.9, we have

$$
\{\mathbf{0}, x, 2 x, \ldots,(\alpha-1) x, \alpha x\} \subseteq \operatorname{dom} f .
$$

The discrete midpoint convexity (2.7) for the pair $(k x,(k+1) x)$ of consecutive points shows

$$
f(k x)+f((k+1) x) \geq f(k x+\lfloor x / 2\rfloor)+f(k x+\lceil x / 2\rceil) \quad(k=0,1, \ldots, \alpha-1) .
$$

Note that $k x$ and $(k+1) x$ are at distance $\|k x-(k+1) x\|_{\infty}=\|x\|_{\infty}$ and therefore, the inequality (2.7) can be used in both global and local cases. The inequality (4.6) implies, in particular, that

$$
k x+\lfloor x / 2\rfloor, k x+\lceil x / 2\rceil \in \operatorname{dom} f \quad(k=0,1, \ldots, \alpha-1) .
$$

By adding the inequalities (4.6) for $k=0,1, \ldots, \alpha-1$ we obtain

$$
f(\mathbf{0})+f(\alpha x) \geq \sum_{k=0}^{\alpha-1}[f(k x+\lfloor x / 2\rfloor)+f(k x+\lceil x / 2\rceil)]-2 \sum_{k=1}^{\alpha-1} f(k x) .
$$

With a parameter $\beta=0,1, \ldots, \alpha-1$ we consider a generalized form of this inequality:

$$
\begin{aligned}
f(\mathbf{0})+f(\alpha x) \geq & \sum_{k=0}^{\alpha-1-\beta}[f(k x+(\beta+1)\lfloor x / 2\rfloor)+f(k x+(\beta+1)\lceil x / 2\rceil)] \\
& -\sum_{k=1}^{\alpha-1-\beta}[f(k x+\beta\lfloor x / 2\rfloor)+f(k x+\beta\lceil x / 2\rceil)] .
\end{aligned}
$$

For $\beta=0$, the inequality $(4.9)$ coincides with $(4.8)$, with each term being finite by $(4.5)$ and (4.7). For $\beta=\alpha-1$, (4.9) coincides with the desired inequality (4.4). We will show the inequality (4.9) for $\beta=0,1, \ldots, \alpha-1$, together with the finiteness of all the terms.

On the right-hand side of (4.9), we classify terms into two parts, $D(\beta)$ and $U(\beta)$, according to whether they contain $\lfloor x / 2\rfloor$ or $\lceil x / 2\rceil$, i.e.,

$$
\begin{aligned}
& D(\beta)=\sum_{k=0}^{\alpha-1-\beta} f(k x+(\beta+1)\lfloor x / 2\rfloor)-\sum_{k=1}^{\alpha-1-\beta} f(k x+\beta\lfloor x / 2\rfloor), \\
& U(\beta)=\sum_{k=0}^{\alpha-1-\beta} f(k x+(\beta+1)\lceil x / 2\rceil)-\sum_{k=1}^{\alpha-1-\beta} f(k x+\beta\lceil x / 2\rceil) .
\end{aligned}
$$

Then (4.9) is expressed as

$$
f(\mathbf{0})+f(\alpha x) \geq D(\beta)+U(\beta) \quad(\beta=0,1, \ldots, \alpha-1) .
$$

This inequality for $\beta=0$ is shown in (4.8). The general case with $\beta \geq 1$ follows from Lemma 4.4 below.

\section{Lemma 4.4.}

(1) $D(\beta) \geq D(\beta+1) \quad(\beta=0,1, \ldots, \alpha-2)$.

(2) $U(\beta) \geq U(\beta+1) \quad(\beta=0,1, \ldots, \alpha-2)$. 
Proof. (1) Consider consecutive terms in the first summation on the right-hand side of (4.10) and note that the $\ell_{\infty}$-distance between $(k x+(\beta+1)\lfloor x / 2\rfloor)$ and $((k+1) x+(\beta+1)\lfloor x / 2\rfloor)$ is equal to $\|x\|_{\infty}$. By applying the discrete midpoint convexity (2.7) to this pair of consecutive terms and using the identity $\lfloor x / 2\rfloor+\lceil x / 2\rceil=x$, we obtain

$$
\begin{aligned}
& f(k x+(\beta+1)\lfloor x / 2\rfloor)+f((k+1) x+(\beta+1)\lfloor x / 2\rfloor) \\
& \geq f(k x+(\beta+1)\lfloor x / 2\rfloor+\lfloor x / 2\rfloor)+f(k x+(\beta+1)\lfloor x / 2\rfloor+\lceil x / 2\rceil) \\
& =f(k x+(\beta+2)\lfloor x / 2\rfloor)+f((k+1) x+\beta\lfloor x / 2\rfloor) \quad(k=0,1, \ldots, \alpha-2-\beta) .
\end{aligned}
$$

We add (4.13) for $k=0,1, \ldots, \alpha-2-\beta$. The sum of the left-hand sides is given by

$$
\begin{aligned}
& \sum_{k=0}^{\alpha-2-\beta} f(k x+(\beta+1)\lfloor x / 2\rfloor)+\sum_{k=0}^{\alpha-2-\beta} f((k+1) x+(\beta+1)\lfloor x / 2\rfloor) \\
& =\sum_{k=0}^{\alpha-1-\beta} f(k x+(\beta+1)\lfloor x / 2\rfloor)+\sum_{k=1}^{\alpha-2-\beta} f(k x+(\beta+1)\lfloor x / 2\rfloor)
\end{aligned}
$$

and the sum of the right-hand sides is given by

$$
\begin{aligned}
& \sum_{k=0}^{\alpha-2-\beta} f(k x+(\beta+2)\lfloor x / 2\rfloor)+\sum_{k=0}^{\alpha-2-\beta} f((k+1) x+\beta\lfloor x / 2\rfloor) \\
& =\sum_{k=0}^{\alpha-2-\beta} f(k x+(\beta+2)\lfloor x / 2\rfloor)+\sum_{k=1}^{\alpha-1-\beta} f(k x+\beta\lfloor x / 2\rfloor) .
\end{aligned}
$$

Then it follows from 4.14$) \geq 4.15)$ that

$$
\begin{aligned}
& \sum_{k=0}^{\alpha-1-\beta} f(k x+(\beta+1)\lfloor x / 2\rfloor)+\sum_{k=1}^{\alpha-2-\beta} f(k x+(\beta+1)\lfloor x / 2\rfloor) \\
& \geq \sum_{k=0}^{\alpha-2-\beta} f(k x+(\beta+2)\lfloor x / 2\rfloor)+\sum_{k=1}^{\alpha-1-\beta} f(k x+\beta\lfloor x / 2\rfloor),
\end{aligned}
$$

which is equivalent to

$$
\begin{aligned}
& \sum_{k=0}^{\alpha-1-\beta} f(k x+(\beta+1)\lfloor x / 2\rfloor)-\sum_{k=1}^{\alpha-1-\beta} f(k x+\beta\lfloor x / 2\rfloor) \\
& \geq \sum_{k=0}^{\alpha-2-\beta} f(k x+(\beta+2)\lfloor x / 2\rfloor)-\sum_{k=1}^{\alpha-2-\beta} f(k x+(\beta+1)\lfloor x / 2\rfloor) .
\end{aligned}
$$

The left-hand side above is equal to $D(\beta)$ and the right-hand side is $D(\beta+1)$. We have thus shown $D(\beta) \geq D(\beta+1)$.

(2) The proof is similar to that of case (1). In place of $(4.13)$ we now have

$$
\begin{aligned}
& f(k x+(\beta+1)\lceil x / 2\rceil)+f((k+1) x+(\beta+1)\lceil x / 2\rceil) \\
& \geq f(k x+(\beta+1)\lceil x / 2\rceil+\lceil x / 2\rceil)+f(k x+(\beta+1)\lceil x / 2\rceil+\lfloor x / 2\rfloor) \\
& =f(k x+(\beta+2)\lceil x / 2\rceil)+f((k+1) x+\beta\lceil x / 2\rceil) \quad(k=0,1, \ldots, \alpha-2-\beta) .
\end{aligned}
$$

In the remaining part, we replace $\lfloor x / 2\rfloor$ by $\lceil x / 2\rceil$.

Theorem 4.2 is analogous to the well-known fact [30] that $\mathrm{L}^{\natural}$-convexity is stable under scaling. In contrast, integral convexity admits the scaling operation only when $n \leq 2$, while for $n \geq 3$, the scaled function $f^{\alpha}$ is not always integrally convex [26, 27]. 


\section{Proximity Theorem}

Let $f: \mathbb{Z}^{n} \rightarrow \mathbb{R} \cup\{+\infty\}$ and $\alpha \in \mathbb{Z}_{++}$. We say that $x^{\alpha} \in \operatorname{dom} f$ is an $\alpha$-local minimizer of $f$ (or $\alpha$-local minimal for $f$ ) if $f\left(x^{\alpha}\right) \leq f\left(x^{\alpha}+\alpha d\right.$ ) for all $d \in\{-1,0,+1\}^{n}$. In general terms a proximity theorem states that for $\alpha \in \mathbb{Z}_{++}$there exists an integer $B(n, \alpha) \in \mathbb{Z}_{+}$such that if $x^{\alpha}$ is an $\alpha$-local minimizer of $f$, then there exists a minimizer $x^{*}$ of $f$ satisfying $\left\|x^{\alpha}-x^{*}\right\|_{\infty} \leq$ $B(n, \alpha)$, where $B(n, \alpha)$ is called the proximity distance.

The following proximity theorem holds for discrete midpoint convex functions. It states that a local minimizer $x^{\alpha}$ of the scaled function $f^{\alpha}(x)=f(\alpha x)$ is close to a minimizer of the original discrete midpoint convex function $f(x)$. Note that $f^{\alpha}$ is discrete midpoint convex by Theorem 4.2, and $x^{\alpha}$ is in fact a global minimizer of $f^{\alpha}$ by Theorems 2.3 and 2.10.

Theorem 5.1. Let $f: \mathbb{Z}^{n} \rightarrow \mathbb{R} \cup\{+\infty\}$ be a (globally or locally) discrete midpoint convex function, $\alpha \in \mathbb{Z}_{++}$, and $x^{\alpha} \in \operatorname{dom} f$. If $f\left(x^{\alpha}\right) \leq f\left(x^{\alpha}+\alpha d\right)$ for all $d \in\{-1,0,+1\}^{n}$, then there exists a minimizer $x^{*} \in \mathbb{Z}^{n}$ of $f$ with $\left\|x^{\alpha}-x^{*}\right\|_{\infty} \leq n(\alpha-1)$.

To prove Theorem 5.1 we may assume $x^{\alpha}=\mathbf{0}$. Define $S=\left\{x \in \mathbb{Z}^{n} \mid\|x\|_{\infty} \leq n(\alpha-1)\right\}$ and $W=\left\{x \in \mathbb{Z}^{n} \mid\|x\|_{\infty}=n(\alpha-1)+1\right\}$, and let $\mu$ be the minimum of $f(x)$ taken over $x \in S$ and $\hat{x}$ be a point in $S$ with $f(\hat{x})=\mu$. We shall show

$$
f(y) \geq \mu \quad \text { for all } y \in W .
$$

Then Theorem 2.4 (box-barrier property) implies that $f(z) \geq \mu$ for all $z \in \mathbb{Z}^{n}$.

Fix $y=\left(y_{1}, \ldots y_{n}\right) \in W$ and let $m=\|y\|_{\infty}$, which is equal to $n(\alpha-1)+1$. With

$$
A_{k}=\left\{i \mid y_{i} \geq m+1-k\right\}, \quad B_{k}=\left\{i \mid y_{i} \leq-k\right\} \quad(k=1, \ldots, m),
$$

we can represent $y$ as

$$
y=\sum_{k=1}^{m}\left(\mathbf{1}_{A_{k}}-\mathbf{1}_{B_{k}}\right) .
$$

We have $A_{1} \subseteq A_{2} \subseteq \cdots \subseteq A_{m}, B_{1} \supseteq B_{2} \supseteq \cdots \supseteq B_{m}, A_{m} \cap B_{1}=\emptyset$, and $A_{1} \cup B_{m} \neq \emptyset$.

Lemma 5.2. There exists some $k_{0} \in\{1,2, \ldots, m-\alpha+1\}$ such that $\left(A_{k_{0}}, B_{k_{0}}\right)=\left(A_{k_{0}+j}, B_{k_{0}+j}\right)$ for $j=1,2, \ldots, \alpha-1$.

Proof. We may assume $A_{1} \neq \emptyset$ by $A_{1} \cup B_{m} \neq \emptyset$ and Propositions 2.13 (3) and 2.14. Define $\left(a_{k}, b_{k}\right)=\left(\left|A_{k}\right|, n-\left|B_{k}\right|\right)$ for $k=1,2, \ldots, m$ and $s=\left|\left\{i \mid y_{i} \geq 1\right\}\right|$. The sequence $\left\{\left(a_{k}, b_{k}\right)\right\}_{k=1,2, \ldots, m}$ is nondecreasing in $\mathbb{Z}^{2}$, satisfying $1 \leq a_{1} \leq a_{2} \leq \cdots \leq a_{m} \leq s$ and $s \leq b_{1} \leq b_{2} \leq \cdots \leq b_{m} \leq n$. That is, $(1, s) \leq\left(a_{1}, b_{1}\right) \leq\left(a_{2}, b_{2}\right) \leq \cdots \leq\left(a_{m}, b_{m}\right) \leq(s, n)$. Since $m=n(\alpha-1)+1$ and the length of a strictly increasing chain connecting $(1, s)$ to $(s, n)$ in $\mathbb{Z}^{2}$ is bounded by $n$, the sequence $\left\{\left(a_{k}, b_{k}\right)\right\}_{k=1,2, \ldots, m}$ must contain, by the pigeonhole principle, a constant subsequence of length $\geq \alpha$. Hence follows the claim.

With reference to the index $k_{0}$ in Lemma 5.2 we define a bipartition $(I, J)$ of $\{1,2, \ldots, m\}$ by $I=\left\{1,2, \ldots, k_{0}-1\right\} \cup\left\{k_{0}+\alpha, k_{0}+\alpha+1, \ldots, m\right\}$ and $J=\left\{k_{0}, k_{0}+1, \ldots, k_{0}+\alpha-1\right\}$. By the parallelogram inequality (3.1) in Theorem 3.1, where $d_{1}=\sum_{i \in I}\left(\mathbf{1}_{A_{i}}-\mathbf{1}_{B_{i}}\right)$ and $d_{2}=$ $\sum_{j \in J}\left(\mathbf{1}_{A_{j}}-\mathbf{1}_{B_{j}}\right)=\alpha d_{0}$ with $d_{0}=\mathbf{1}_{A_{k_{0}}}-\mathbf{1}_{B_{k_{0}}}$, we obtain

$$
f(\mathbf{0})+f(y) \geq f\left(d_{1}\right)+f\left(\alpha d_{0}\right) .
$$


Here we have $f\left(\alpha d_{0}\right) \geq f(\mathbf{0})$ by the $\alpha$-local minimality of $\mathbf{0}$. We also have $d_{1} \in S$ since

$$
\left\|d_{1}\right\|_{\infty}=\left\|\sum_{i \in I}\left(\mathbf{1}_{A_{i}}-\mathbf{1}_{B_{i}}\right)\right\|_{\infty}=|I|=m-\alpha=(n-1)(\alpha-1) \leq n(\alpha-1),
$$

and hence $f\left(d_{1}\right) \geq \mu$ by the definition of $\mu$. Therefore,

$$
f(y) \geq f\left(d_{1}\right)+\left[f\left(\alpha d_{0}\right)-f(\mathbf{0})\right] \geq \mu+0=\mu .
$$

This establishes (5.1), completing the proof of Theorem 5.1.

Theorem 5.1 is a generalization of the proximity theorem for $\mathrm{L}^{\natural}$-convex functions below. We have the same proximity bound $n(\alpha-1)$ in both theorems.

Theorem $5.3([20,30])$. Let $f: \mathbb{Z}^{n} \rightarrow \mathbb{R} \cup\{+\infty\}$ be an $\mathrm{L}^{\natural}$-convex function, $\alpha \in \mathbb{Z}_{++}$, and $x^{\alpha} \in \operatorname{dom} f$. If $f\left(x^{\alpha}\right) \leq f\left(x^{\alpha}+\alpha d\right)$ for all $d \in\{0,1\}^{n} \cup\{0,-1\}^{n}$, then there exists a minimizer $x^{*}$ of $f$ with $\left\|x^{\alpha}-x^{*}\right\|_{\infty} \leq n(\alpha-1)$.

The following example demonstrates the tightness of the bound in Theorems 5.1 and 5.3 . This example also shows that the bound $n(\alpha-1)$ is tight for a linear function defined on a simple polyhedron.

Example 5.1 ([26, 27, 35]). Let $X=\left\{x \in \mathbb{Z}^{n} \mid 0 \leq x_{i}-x_{i+1} \leq \alpha-1(i=1, \ldots, n-\right.$ $\left.1), 0 \leq x_{n} \leq \alpha-1\right\}$. The function $f$ defined by $f(x)=-x_{1}$ on $\operatorname{dom} f=X$ is an $\mathrm{L}^{\natural}-$ convex (and hence discrete midpoint convex) function and has a unique minimizer at $x^{*}=$ $(n(\alpha-1),(n-1)(\alpha-1), \ldots, 2(\alpha-1), \alpha-1)$. On the other hand, $x^{\alpha}=\mathbf{0}$ is $\alpha$-local minimal, since $X \cap\{-\alpha, 0, \alpha\}^{n}=\{\mathbf{0}\}$. We have $\left\|x^{\alpha}-x^{*}\right\|_{\infty}=n(\alpha-1)$, which shows the tightness of the bound $n(\alpha-1)$ given in Theorem 5.3. This example is given in [26, 27] as a reformulation of [35, Remark 2.3] for L-convex functions to $\mathrm{L}^{\natural}$-convex functions.

For (general) integrally convex functions, in contrast, the proximity bound $n(\alpha-1)$ is valid only when $n \leq 2$, and a quadratic lower bound $(n-2)^{2}(\alpha-1) / 4$ for the proximity distance is demonstrated in [26, 27] (no better lower bound is known). The following proximity theorem, with a superexponential proximity bound, is known for integrally convex functions.

Theorem 5.4 ([26, 27]). Let $f: \mathbb{Z}^{n} \rightarrow \mathbb{R} \cup\{+\infty\}$ be an integrally convex function, $\alpha \in \mathbb{Z}_{++}$, and $x^{\alpha} \in \operatorname{dom} f$. If $f\left(x^{\alpha}\right) \leq f\left(x^{\alpha}+\alpha d\right)$ for all $d \in\{-1,0,+1\}^{n}$, then there exists a minimizer $x^{*}$ of $f$ with $\left\|x^{\alpha}-x^{*}\right\|_{\infty} \leq \beta_{n}(\alpha-1)$, where $\beta_{1}=1, \beta_{2}=2$, and $\beta_{n} \leq(n+1) ! / 2^{n-1}(n=3,4, \ldots)$.

\section{Minimization Algorithms}

\subsection{2-neighborhood steepest descent algorithm}

In this section we propose a variant of the descent algorithm that is suited for discrete midpoint convex functions. The algorithm repeats finding a local minimizer in the neighborhood of $\ell_{\infty}$-distance 2 , and is named the 2-neighborhood steepest descent algorithm.

Let $f: \mathbb{Z}^{n} \rightarrow \mathbb{R} \cup\{+\infty\}$ be a (locally or globally) discrete midpoint convex function with $\arg \min f \neq \emptyset$. Fix any

$$
x^{(0)} \in \operatorname{dom} f \backslash \arg \min f
$$


and denote the minimum $\ell_{\infty}$-distance to a minimizer of $f$ by

$$
L=L\left(x^{(0)}\right)=\min \left\{\left\|x-x^{(0)}\right\|_{\infty} \mid x \in \arg \min f\right\} .
$$

For $k=2,3, \ldots, L$, define

$$
S_{k}=S_{k}\left(x^{(0)}\right)=\left\{x \in \mathbb{Z}^{n} \mid\left\|x-x^{(0)}\right\|_{\infty} \leq k\right\} .
$$

The idea of our algorithm is to generate a sequence of points that minimize $f$ within $S_{k}$ for $k=2,3, \ldots, L$. The following proposition states that the consecutive points can be chosen to be close to each other, at $\ell_{\infty}$-distance less than or equal to 2 .

Proposition 6.1. Assume $3 \leq k \leq L$. For any $x^{(k-1)} \in \arg \min \left\{f(x) \mid x \in S_{k-1}\right\}$, there exists $x^{(k)} \in \arg \min \left\{f(x) \mid x \in S_{k}\right\}$ that satisfies $\left\|x^{(k)}-x^{(k-1)}\right\|_{\infty} \leq 2$.

Proof. Let $y$ be an element of $\arg \min \left\{f(x) \mid x \in S_{k}\right\}$ with $\left\|y-x^{(k-1)}\right\|_{\infty}$ minimum, and put $m=\left\|y-x^{(k-1)}\right\|_{\infty}$. We can prove $m \leq 2$ (see below). Then we can take $x^{(k)}=y$.

To prove $m \leq 2$ by contradiction, suppose that $m \geq 3$. We consider the decomposition of $y-x^{(k-1)}$ in the form of 2.5 with $A_{j}=\left\{i \mid y_{i}-x_{i}^{(k-1)} \geq m+1-j\right\}$ and $B_{j}=\left\{i \mid y_{i}-x_{i}^{(k-1)} \leq-j\right\}$ for $j=1,2, \ldots, m$. Let $d=\mathbf{1}_{A_{2}}-\mathbf{1}_{B_{2}}$. By the parallelogram inequality (3.4) in Theorem 3.2, we obtain

$$
f\left(x^{(k-1)}\right)+f(y) \geq f\left(x^{(k-1)}+d\right)+f(y-d) .
$$

Here we have

$$
x^{(k-1)}+d \in S_{k-1}, \quad y-d \in S_{k},
$$

which are shown in Claim below. Since $x^{(k-1)}$ is a minimizer in $S_{k-1}$, the former implies $f\left(x^{(k-1)}+d\right) \geq f\left(x^{(k-1)}\right)$. The latter implies the strict inequality $f(y-d)>f(y)$ by $\|(y-d)-$ $x^{(k-1)}\left\|_{\infty}<\right\| y-x^{(k-1)} \|_{\infty}$ and the definition of $y$. The addition of these two inequalities yields a contradiction to 6.3 .

\section{Claim.}

(i) $\left(x_{i}^{(k-1)}+1\right)-x_{i}^{(0)} \leq k-1$ for $i \in A_{2}$.

(ii) $\left(x_{i}^{(k-1)}-1\right)-x_{i}^{(0)} \geq-(k-1)$ for $i \in B_{2}$.

(iii) $\left(y_{i}-1\right)-x_{i}^{(0)} \geq-k$ for $i \in A_{2}$.

(iv) $\left(y_{i}+1\right)-x_{i}^{(0)} \leq k$ for $i \in B_{2}$.

(Proof) (i) By $y \in S_{k}, i \in A_{2}$, and $m \geq 3$ we have $x_{i}^{(0)}+k \geq y_{i} \geq x_{i}^{(k-1)}+(m-1) \geq x_{i}^{(k-1)}+2$.

(ii) By $y \in S_{k}$ and $i \in B_{2}$ we have $x_{i}^{(0)}-k \leq y_{i} \leq x_{i}^{(k-1)}-2$.

(iii) By $x^{(k-1)} \in S_{k-1}, i \in A_{2}$, and $m \geq 3$ we have $x_{i}^{(0)}-(k-1) \leq x_{i}^{(k-1)} \leq y_{i}-(m-1) \leq y_{i}-2$.

(iv) By $x^{(k-1)} \in S_{k-1}$ and $i \in B_{2}$ we have $x_{i}^{(0)}+(k-1) \geq x_{i}^{(k-1)} \geq y_{i}+2$.

On the basis of Proposition 6.1 we can devise an algorithm which generates a sequence of points $\left\{x^{(k)}\right\}_{k}$ such that $x^{(k)}$ is a minimizer of $f$ in $S_{k}\left(x^{(0)}\right)$ and is at $\ell_{\infty}$-distance at most two from the preceding point $x^{(k-1)}$. For $x \in \mathbb{Z}^{n}$ we define

$$
N_{2}(x)=\left\{y \in \mathbb{Z}^{n} \mid\|y-x\|_{\infty} \leq 2\right\}
$$

and call it the 2-neighborhood of $x$. We assume that an oracle is available which finds, for any $x \in \operatorname{dom} f$, a minimizer of $f$ in $N_{2}(x)$ intersected with a box. We refer to such an oracle as a 2-neighborhood minimization oracle. We also assume that an initial point $x^{(0)}$ in $\operatorname{dom} f$ can be found (or is given). 


\section{The 2-neighborhood steepest descent algorithm}

D0: Find $x^{(0)} \in \operatorname{dom} f$, find $x^{(2)}$ that minimizes $f(x)$ in $S_{2}\left(x^{(0)}\right)$, and set $k:=3$.

D1: Find $x^{(k)}$ that minimizes $f(x)$ in $N_{2}\left(x^{(k-1)}\right) \cap S_{k}\left(x^{(0)}\right)$.

D2: If $f\left(x^{(k)}\right)=f\left(x^{(k-1)}\right)$, then output $x^{(k-1)}$ and stop.

D3: Set $k:=k+1$, and go to D1.

The following theorem states that this algorithm finds a minimizer of $f$ in $L$ iterations. It is emphasized that we do not have to know $L$ in advance, but we can obtain $L$ as an outcome of the algorithm.

Theorem 6.2. For a (globally or locally) midpoint convex function $f$ with $\arg \min f \neq \emptyset$, the 2-neighborhood steepest descent algorithm finds a minimizer of $f$ in L iterations.

Proof. By Proposition 6.1 the point $x^{(k)}$ generated by the algorithm is in fact a minimizer of $f$ in $S_{k}\left(x^{(0)}\right)$. That is, we have

$$
x^{(k)} \in \arg \min \left\{f(x) \mid x \in S_{k}\left(x^{(0)}\right)\right\} \quad(k=2,3, \ldots) .
$$

Claim. If $f\left(x^{(k)}\right)=f\left(x^{(k-1)}\right)$ in Step D2, then $x^{(k-1)}$ is a minimizer of $f$.

(Proof) For any $d \in\{-1,0,+1\}^{n}, x^{(k-1)}+d$ belongs to $S_{k}\left(x^{(0)}\right)$, and hence $f\left(x^{(k)}\right) \leq f\left(x^{(k-1)}+d\right)$ by (6.4). Therefore, if $f\left(x^{(k)}\right)=f\left(x^{(k-1)}\right)$, then $f\left(x^{(k-1)}\right) \leq f\left(x^{(k-1)}+d\right)$ for any $d$. This implies $x^{(k-1)} \in \arg \min f$ by Theorem 2.3, since $f$ is integrally convex by Theorem 2.10. (End of the proof of Claim)

By the definition 6.1 of $L, S_{k}\left(x^{(0)}\right)$ contains a minimizer of $f$ if and only if $k \geq L$. In particular, $x^{(L)}$ is a minimizer of $f$ and $f\left(x^{(L)}\right)=f\left(x^{(L+1)}\right)$, whereas $f\left(x^{(k)}\right) \neq f\left(x^{(k-1)}\right)$ for any $k \leq L$. Therefore, the number of iterations of the above algorithm is equal to $L$.

Let $F(n)$ denote the number of function evaluations needed by the 2-neighborhood minimization oracle. Since $N_{2}(x)$ consists of $5^{n}$ points, $F(n)$ is bounded by $5^{n}$. However, it can be smaller for a subclass of discrete midpoint convex functions; for example, $F(n)$ is polynomial in $n$ for $\mathrm{L}^{\natural}$-convex functions. It is also noted that $F(n)$ cannot be polynomial in $n$ for general discrete midpoint convex functions, since every function on the unit cube $\{0,1\}^{n}$ is trivially discrete midpoint convex. By Theorem 6.2, the total number of function evaluations of the above algorithm is $\mathrm{O}(F(n) L)$, which is bounded by $\mathrm{O}\left(5^{n} L\right)$.

Remark 6.1. Theorem 6.2 shows that the sequence of points generated by the 2-neighborhood steepest descent algorithm connects the initial point $x^{(0)}$ and a nearest minimizer of $f$ with $L-1$ intermediate points, where $L$ is the minimum $\ell_{\infty}$-distance from the initial point $x^{(0)}$ to a minimizer of $f$. Similar facts are pointed out for $\mathrm{L}^{\natural}$-convex function minimization algorithms by Kolmogorov and Shioura [23] and Murota and Shioura [33]; see also Shioura [37].

\subsection{Scaling algorithm}

The scaling property (Theorem 4.2) and the proximity theorem (Theorem 5.1p enable us to design a scaling algorithm for the minimization of (globally or locally) discrete midpoint convex functions with bounded effective domains. The proposed algorithm employs the standard proximity-scaling framework while using the 2-neighborhood steepest descent algorithm of Section 6.1 as a subroutine.

For each scaling unit $\alpha$, we minimize the scaled function $f^{\alpha}(y)=f(x+\alpha y)$ with the origin at the current $x$, find a minimizer $y$ of $f^{\alpha}(y)$ by the 2-neighborhood steepest descent algorithm 
of Section 6.1, and update $x$ to $x+\alpha y$. It is emphasized that the scaled function $f^{\alpha}$ is discrete midpoint convex by Theorem 4.2 and it has a minimizer $y$ with the magnitude $\|y\|_{\infty}$ controlled by the proximity result in Theorem 5.1. Recall that $K_{\infty}$ denotes the $\ell_{\infty}$-size of the effective domain of $f$, i.e., $K_{\infty}=\max \left\{\|x-y\|_{\infty} \mid x, y \in \operatorname{dom} f\right\}$, where we assume $K_{\infty}>0$ to avoid triviality.

\section{Scaling algorithm for discrete midpoint convex functions}

S0: Find a vector $x \in \operatorname{dom} f$ and set $\alpha:=2^{\left\lceil\log _{2}\left(K_{\infty}+1\right)\right\rceil}$.

S1: Find a vector $y$ that minimizes $f^{\alpha}(y)=f(x+\alpha y)$ subject to the constraint $\|y\|_{\infty} \leq n$ (by the 2-neighborhood steepest descent algorithm), and set $x:=x+\alpha y$.

S2: If $\alpha=1$, then stop ( $x$ is a minimizer of $f$ ).

S3: Set $\alpha:=\alpha / 2$, and go to S1.

The correctness of the algorithm can be shown as follows. Let $x^{2 \alpha}$ denote the vector $x$ at the beginning of Step S1 for $\alpha$. The function $f^{\alpha}(y)=f\left(x^{2 \alpha}+\alpha y\right)$ is discrete midpoint convex by Theorem 4.2. Let $y^{\alpha}$ be the vector computed in Step S1 and $x^{\alpha}=x^{2 \alpha}+\alpha y^{\alpha}$. The proximity theorem (Theorem 5.1) applied to $f^{\alpha}$ guarantees that if $y=\mathbf{0}$ is 2-local minimal for $f^{\alpha}$, then $y^{\alpha}$ is a (global) minimizer of $f^{\alpha}$. In other words, if $x^{2 \alpha}$ is $2 \alpha$-local minimal for $f$, then $x^{\alpha}$ is $\alpha$-local minimal for $f$. At the beginning of the algorithm we have $\alpha=2^{\left\lceil\log _{2}\left(K_{\infty}+1\right)\right\rceil}$, and hence the initial vector $x=x^{2 \alpha}$ is obviously $2 \alpha$-local minimal for $f$. At the termination of the algorithm we have $\alpha=1$, and the output of the algorithm is indeed a minimizer of $f$, since 1-local minimality for discrete midpoint convex functions is the same as the global minimality by Theorems 2.3 and 2.10 .

The complexity of the algorithm can be analyzed as follows. By Theorem 6.2, Step S1 can be done with at most $n$ calls of the 2-neighborhood minimization oracle. Since the number of scaling phases is $\log _{2} K_{\infty}$, the total number of oracle calls is $\mathrm{O}\left(n \log _{2} K_{\infty}\right)$. As for Step S0, we assume that an initial point $x$ and the size $K_{\infty}$ are available, since we have no general efficient method for computing them. With this understanding we obtain the following theorem. Recall that $F(n)$ denotes the number of function evaluations needed by the 2-neighborhood minimization oracle.

Theorem 6.3. For a (globally or locally) midpoint convex function $f$ with a bounded effective domain $\operatorname{dom} f$, the scaling algorithm finds a minimizer of $f$ with $\mathrm{O}\left(n F(n) \log _{2} K_{\infty}\right)$ function evaluations.

The algorithm runs with $\mathrm{O}\left(C(n) \log _{2} K_{\infty}\right)$ function evaluations for $C(n)=n F(n)$. This means that, if the dimension $n$ is fixed, the algorithm is polynomial in the problem size. With the naive bound $F(n) \leq 5^{n}$, we obtain $C(n) \leq 5^{n} n$ and the complexity of the scaling algorithm above is $\mathrm{O}\left(5^{n} n \log _{2} K_{\infty}\right)$. It is noted that no algorithm can be polynomial in $n$ for general discrete midpoint convex functions, since every function on the unit cube $\{0,1\}^{n}$ is trivially discrete midpoint convex.

Our algorithm has much less complexity than the scaling algorithm for general integrally convex functions developed in [27]. The number of function evaluations of the algorithm of [27] is known to be bounded by $\mathrm{O}\left(C_{0}(n) \log _{2} K_{\infty}\right)$ with $C_{0}(n)=\left(12(n+1) ! / 2^{n-1}\right)^{n}$, which is much larger than $C(n) \leq 5^{n} n$ of our algorithm for discrete midpoint convex functions.

\section{Quadratic Functions}

In this section we study discrete midpoint convexity of a quadratic function $f(x)=x^{\top} Q x$, where $Q$ is an $n \times n$ symmetric matrix and $x \in \mathbb{Z}^{n}$. We start with a general characterization of 
(locally and globally) discrete midpoint convex quadratic functions.

Lemma 7.1. For $f(x)=x^{\top} Q x$, the discrete midpoint convexity 2.7) for $x, y \in \mathbb{Z}^{n}$ is equivalent to

$$
z^{\top} Q z \geq \mathbf{1}_{J}^{\top} Q \mathbf{1}_{J}
$$

for $z=x-y$, where $J=\left\{i \mid z_{i}\right.$ is odd $\}$. Hence, $f$ is locally (resp., globally) discrete midpoint convex if and only if (7.1) holds for all $z \in \mathbb{Z}^{n}$ with $\|z\|_{\infty}=2$ (resp., $\|z\|_{\infty} \geq 2$ ).

Proof. With the use of identities

$$
\begin{aligned}
& \frac{1}{2}\left(\left\lfloor\frac{x+y}{2}\right\rfloor+\left[\frac{x+y}{2}\right\rceil\right)=\frac{x+y}{2}, \\
& f(x)+f(y)-2 f\left(\frac{x+y}{2}\right)=\frac{1}{2}(x-y)^{\top} Q(x-y),
\end{aligned}
$$

we can rewrite the discrete midpoint convexity $(2.7)$ to

$$
(x-y)^{\top} Q(x-y) \geq\left(\left\lceil\frac{x+y}{2}\right\rceil-\left\lfloor\frac{x+y}{2}\right\rfloor\right)^{\top} Q\left(\left\lceil\frac{x+y}{2}\right\rceil-\left\lfloor\frac{x+y}{2}\right\rfloor\right) .
$$

The substitution of $x-y=z$ and $\left\lceil\frac{x+y}{2}\right\rceil-\left\lfloor\frac{x+y}{2}\right\rfloor=\mathbf{1}_{J}$ yields 7.1 .

It follows from Lemma 7.1 and Proposition 3.3 (3) that the set of matrices $Q$ for which $f(x)=x^{\top} Q x$ is (locally or globally) discrete midpoint convex is a polyhedral convex cone.

We next consider the relationship between the discrete midpoint convexity of $f(x)=$ $x^{\top} Q x$ and the diagonal dominance ${ }^{7}$ of $Q$ :

$$
q_{i i} \geq \sum_{j \neq i}\left|q_{i j}\right| \quad(i=1, \ldots, n)
$$

The following facts are known for integral convexity and $\mathrm{L}^{\natural}$-convexity.

Proposition 7.2 ([5, Proposition 4.5, Remark 4.3]). If $Q$ is diagonally dominant (7.2), then $f(x)=x^{\top} Q x$ is integrally convex. The converse is also true if $n \leq 2$.

Proposition 7.3 ([30, Section 7.3]). $f(x)=x^{\top} Q x$ is $\mathrm{L}^{\natural}$-convex if and only if $Q$ is diagonally dominant $\left(7.2\right.$, and $q_{i j} \leq 0$ for all $i \neq j$.

In contrast, discrete midpoint convexity is not governed by diagonal dominance. This is demonstrated in the following examples.

Example 7.1. Let $f(x)=x^{\top} Q x$ with $Q=\left[\begin{array}{rrr}1 & -1 & 1 \\ -1 & 2 & -1 \\ 1 & -1 & 2\end{array}\right]$ for $x \in \mathbb{Z}^{3}$. The matrix $Q$ is not diagonally dominant. Nevertheless, the function $f(x)$ is globally (and hence locally) midpoint convex. Indeed, it is possible to verify the inequality 7.1 in Lemma 7.1 for all $z \in \mathbb{Z}^{3}$ with $\|z\|_{\infty} \geq 2$.

\footnotetext{
${ }^{7}$ To be precise, the condition 7.2 says that $Q$ is a diagonally dominant matrix with nonnegative diagonals. In this paper, however, we refer to $(7.2)$ simply as diagonal dominance.
} 
Example 7.2. Let $f(x)=x^{\top} Q x$ with $Q=\left[\begin{array}{lll}1 & 1 & 0 \\ 1 & 1 & 0 \\ 0 & 0 & 0\end{array}\right]$ for $x \in \mathbb{Z}^{3}$. The matrix $Q$ is diagonally dominant. Nevertheless, the function $f(x)=\left(x_{1}+x_{2}\right)^{2}$ is not locally midpoint convex in $\left(x_{1}, x_{2}, x_{3}\right)$. Indeed, for $x=(1,0,0)$ and $y=(0,1,2)$, we have $z=\left\lceil\frac{x+y}{2}\right\rceil=(1,1,1), w=$ $\left\lfloor\frac{x+y}{2}\right\rfloor=(0,0,1)$, and $f(x)+f(y)=1+1<f(z)+f(w)=4+0$. It is also noted that the function $\left(x_{1}+x_{2}\right)^{2}$ is locally midpoint convex in $\left(x_{1}, x_{2}\right)$.

We can still make the following statement.

Proposition 7.4. If $f$ is locally discrete midpoint convex and $q_{i j} \leq 0$ for all $i \neq j$, then $Q$ is diagonally dominant.

Proof. Since $f$ is locally discrete midpoint convex, it is integrally convex by Theorem 2.10 . Furthermore it is submodular by $q_{i j} \leq 0(i \neq j)$, and hence $\mathrm{L}^{\natural}$-convex by Theorem 2.8 . Finally Proposition 7.3 shows the diagonal dominance of $Q$.

For two-dimensional quadratic functions, local and global discrete midpoint convexities can be characterized as follows.

Proposition 7.5. For $n=2, f(x)=x^{\top} Q x$ is locally discrete midpoint convex if and only if $q_{11} \geq\left|q_{12}\right|$ and $q_{22} \geq\left|q_{12}\right|$.

Proof. Suppose that $f$ is locally discrete midpoint convex. By inequality $(7.1)$ for $z=(2,1)$ and $z=(2,-1)$ we obtain $q_{11}+q_{12} \geq 0$ and $q_{11}-q_{12} \geq 0$, i.e., $q_{11}-\left|q_{12}\right| \geq 0$. By symmetry, we also have $q_{22}-\left|q_{21}\right| \geq 0$. Conversely, these conditions imply (7.1) for all $z$ with $\|z\|_{\infty}=2$.

Proposition 7.6. For $n=2, f(x)=x^{\top} Q x$ is globally discrete midpoint convex if and only if $q_{11} \geq\left|q_{12}\right|, q_{22} \geq\left|q_{12}\right|$, and $q_{11}+q_{22} \geq(5 / 2) q_{12}$.

Proof. Suppose that $f$ is globally discrete midpoint convex. By inequality (7.1) for $z=$ $(3,-3)$ we obtain $2\left(q_{11}+q_{22}\right) \geq 5 q_{12}$. Using also Proposition 7.5 we obtain three inequalities $q_{11} \geq\left|q_{12}\right|, q_{22} \geq\left|q_{12}\right|$, and $q_{11}+q_{22} \geq(5 / 2) q_{12}$. Conversely, these conditions imply (7.1) for all $z=(u, v)$ with $\max (|u|,|v|) \geq 2$, as follows.

Inequality (7.1) for $z=(u, v)$ is easy to see if $u$ or $v$ is even. For example, if $u$ odd and $v$ even, 7.1 is equivalent to $q_{11} u^{2}+q_{22} v^{2}+2 q_{12} u v \geq q_{11}$, which can be shown as follows:

$$
\begin{aligned}
q_{11} u^{2}+q_{22} v^{2}+2 q_{12} u v & \geq q_{11} u^{2}+q_{22} v^{2}-2\left|q_{12}\right||u v| \\
& =\left|q_{12}\right|(|u|-|v|)^{2}+\left(q_{11}-\left|q_{12}\right|\right) u^{2}+\left(q_{22}-\left|q_{12}\right|\right) v^{2} \\
& \geq\left|q_{12}\right|+\left(q_{11}-\left|q_{12}\right|\right)=q_{11},
\end{aligned}
$$

where the second inequality follows from $|u|-|v| \neq 0$ and $u \neq 0$.

Assume that $u$ and $v$ are odd. Then

$$
\begin{aligned}
\text { (7.1) } & \Leftrightarrow q_{11} u^{2}+q_{22} v^{2}+2 q_{12} u v \geq q_{11}+q_{22}+2 q_{12} \\
& \Leftrightarrow F:=q_{11}\left(u^{2}-1\right)+q_{22}\left(v^{2}-1\right)+2 q_{12}(u v-1) \geq 0 .
\end{aligned}
$$

We consider the following five cases separately:

Case $1:|u| \geq 3$ and $|v|=1$,

Case 2: $|v| \geq 3$ and $|u|=1$,

Case 3: $|u| \geq 3,|v| \geq 3$, and $q_{12} u v \geq 0$,

Case 4: $|u| \geq 3,|v| \geq 3, q_{12} \geq 0$, and $u v<0$.

Case 5: $|u| \geq 3,|v| \geq 3, q_{12} \leq 0$, and $u v>0$. 
Case $1(|u| \geq 3$ and $|v|=1)$ : We have

$$
F \geq q_{11}\left(u^{2}-1\right)-2\left|q_{12}\right|(|u|+1) \geq q_{11}\left(u^{2}-1\right)-2 q_{11}(|u|+1)=q_{11}\left((|u|-1)^{2}-4\right) \geq 0 .
$$

Case $2(|v| \geq 3$ and $|u|=1)$ : Similarly to Case 1 .

Case $3\left(|u| \geq 3,|v| \geq 3, q_{12} u v \geq 0\right)$ : We have $q_{12}(u v-1) \geq 0$ in this case, and hence

$$
F=q_{11}\left(u^{2}-1\right)+q_{22}\left(v^{2}-1\right)+2 q_{12}(u v-1) \geq q_{11}\left(u^{2}-1\right)+q_{22}\left(v^{2}-1\right) \geq 0 .
$$

Case $4\left(|u| \geq 3,|v| \geq 3, q_{12} \geq 0, u v<0\right)$ : We have

$$
\begin{aligned}
F & =q_{11}\left(u^{2}-1\right)+q_{22}\left(v^{2}-1\right)-2 q_{12}(|u v|+1) \\
& =q_{12}(|u|-|v|)^{2}+\left(q_{11}-q_{12}\right) u^{2}+\left(q_{22}-q_{12}\right) v^{2}-q_{11}-q_{22}-2 q_{12} \\
& \geq\left(q_{11}-q_{12}\right) \times 9+\left(q_{22}-q_{12}\right) \times 9-q_{11}-q_{22}-2 q_{12} \\
& =8\left(q_{11}+q_{22}\right)-20 q_{12} \geq 0 .
\end{aligned}
$$

Case $5\left(|u| \geq 3,|v| \geq 3, q_{12} \leq 0, u v>0\right)$ : We have

$$
\begin{aligned}
F & =q_{11}\left(u^{2}-1\right)+q_{22}\left(v^{2}-1\right)-2\left|q_{12}\right|(u v-1) \\
& =\left|q_{12}\right|(u-v)^{2}+\left(q_{11}-\left|q_{12}\right|\right) u^{2}+\left(q_{22}-\left|q_{12}\right|\right) v^{2}-q_{11}-q_{22}+2\left|q_{12}\right| \\
& \geq\left(q_{11}-\left|q_{12}\right|\right) \times 9+\left(q_{22}-\left|q_{12}\right|\right) \times 9-q_{11}-q_{22}+2\left|q_{12}\right| \\
& =8\left(q_{11}-\left|q_{12}\right|\right)+8\left(q_{22}-\left|q_{12}\right|\right) \geq 0 .
\end{aligned}
$$

For the general $n$-dimensional case, we have the following sufficient condition for global discrete midpoint convexity of quadratic functions. The minimum and maximum eigenvalues of $Q$ are denoted, respectively, by $\lambda_{\min }^{Q}$ and $\lambda_{\max }^{Q}$.

Proposition 7.7. $f(x)=x^{\top} Q x$ is globally discrete midpoint convex if

$$
\lambda_{\min }^{Q} \geq \frac{n-1}{n+3} \lambda_{\max }^{Q}
$$

Proof. We prove that (7.3) implies (7.1). We may assume $n \geq 2$.

For an $n \times n$ symmetric matrix $C$, let $\lambda_{\min }^{C}$ and $\lambda_{\max }^{C}$ denote its minimum and maximum eigenvalues, respectively. Furthermore, for a set $I \subseteq N=\{1, \ldots, n\}$ of indices, let $C_{I}$ denote the submatrix of $C$ with row and column indices in $I$. Note that (7.3) implies

$$
\lambda_{\min }^{Q_{I}} \geq \frac{n-1}{n+3} \lambda_{\max }^{Q_{I}}
$$

for any $I \subseteq N$, since $0 \leq \lambda_{\min }^{Q} \leq \lambda_{\min }^{Q_{I}} \leq \lambda_{\max }^{Q_{I}} \leq \lambda_{\max }^{Q}$.

Take $z \in \mathbb{Z}^{n}$ with $\|z\|_{\infty} \geq 2$, and let $I=\left\{i \mid z_{i} \neq 0\right\}$ and $m=|I|$, whereas $J=\left\{i \mid z_{i}\right.$ is odd $\}$. If $\|z\|_{\infty}=2$, we have $z^{\top} z \geq 2^{2}+(|I|-1)=m+3$ and $\mathbf{1}_{J}^{\top} \mathbf{1}_{J}=|J| \leq m-1$. If $\|z\|_{\infty} \geq 3$, we have $z^{\top} z \geq 3^{2}+(|I|-1)=m+8$ and $\mathbf{1}_{J}^{\top} \mathbf{1}_{J}=|J| \leq m$. That is, by defining

$$
(\zeta, \eta)= \begin{cases}(m+3, m-1) & \left(\text { if }\|z\|_{\infty}=2\right) \\ (m+8, m) & \left(\text { if }\|z\|_{\infty} \geq 3\right)\end{cases}
$$


we have $z^{\top} z \geq \zeta$ and $\mathbf{1}_{J}^{\top} \mathbf{1}_{J} \leq \eta$. Then (7.1) can be shown as follows:

$$
\begin{aligned}
z^{\top} Q z & \geq \zeta \frac{z^{\top} Q z}{z^{\top} z} \geq \zeta \lambda_{\min }^{Q_{I}} \geq \zeta \frac{n-1}{n+3} \lambda_{\max }^{Q_{I}} \\
& \geq \zeta \frac{n-1}{n+3} \frac{\mathbf{1}_{J}^{\top} Q \mathbf{1}_{J}}{\mathbf{1}_{J}^{\top} \mathbf{1}_{J}} \geq \frac{\zeta}{\eta} \frac{n-1}{n+3} \mathbf{1}_{J}^{\top} Q \mathbf{1}_{J} \geq \mathbf{1}_{J}^{\top} Q \mathbf{1}_{J},
\end{aligned}
$$

where the six inequalities follow, respectively, from: $z^{\top} z \geq \zeta>0$, the definition of $I$, (7.4), $I \supseteq J, \mathbf{1}_{J}^{\top} \mathbf{1}_{J} \leq \eta$, and $\zeta(n-1) \geq \eta(n+3)$. (Proof of $\zeta(n-1) \geq \eta(n+3)$ : if $\|z\|_{\infty}=2$, we have $\zeta(n-1)-\eta(n+3)=(m+3)(n-1)-(m-1)(n+3)=4(n-m) \geq 0$; and if $\|z\|_{\infty} \geq 3$, we have $\zeta(n-1)-\eta(n+3)=(m+8)(n-1)-m(n+3)=4(2 n-m-2) \geq 4(n-2) \geq 0$.

The converse of Proposition 7.7 is not true, which is shown by the following example.

Example 7.3. The matrix $Q$ in Example 7.1, yielding a globally midpoint convex function, does not satisfy the condition $(7.3)$ in Proposition 7.7. The three eigenvalues of $Q$ are given by $2-\sqrt{3}=\lambda_{\min }^{Q}, 1$, and $2+\sqrt{3}=\lambda_{\max }^{Q}$, for which the left-hand side of 7.3 is equal to $2-\sqrt{3}=0.268 \cdots$ and the right-hand side to $(2+\sqrt{3}) / 3=1.244 \cdots$.

We note the following as a corollary of Proposition 7.7.

Proposition 7.8. Assume $n \geq 2$. $f(x)=x^{\top} Q x$ is globally discrete midpoint convex if $Q$ is represented as $Q=\alpha(I+R)$ with $\alpha \geq 0$ and a positive semidefinite $R$ satisfying

$$
\max _{1 \leq i \leq n} \sum_{j=1}^{n}\left|r_{i j}\right| \leq \frac{4}{n-1} .
$$

Proof. We have $\lambda_{\max }^{Q}=\alpha\left(1+\lambda_{\max }^{R}\right)$ and $\lambda_{\max }^{R} \leq \max _{1 \leq i \leq n} \sum_{j=1}^{n}\left|r_{i j}\right|$, where the latter is a consequence of the Gershgorin theorem. Hence 7.5 implies $\lambda_{\max }^{Q} \leq \alpha(n+3) /(n-1)$. On the other hand, we have $\lambda_{\min }^{Q} \geq \alpha$ since $R$ is positive semidefinite. Hence follows 7.3 .

\section{Proofs about Discrete Midpoint Convex Sets}

\subsection{Proof of Proposition 2.5 (2)}

Let $S \subseteq \mathbb{Z}^{n}$ be a discrete midpoint convex set. We prove that $S$ is an integrally convex set. That is, we are to show that $z \in \bar{S}$ implies $z \in \overline{S \cap N(z)}$. Fix any $z \in \bar{S}$, which can be represented as a convex combination

$$
z=\sum_{i=1}^{r} \lambda_{i} y^{i}, \quad \sum_{i=1}^{r} \lambda_{i}=1, \quad \lambda_{i}>0(i=1,2, \ldots, r)
$$

of some $y^{1}, y^{2}, \ldots, y^{r} \in S$ with coefficients $\lambda_{1}, \lambda_{2}, \ldots, \lambda_{r} \in \mathbb{R}$.

With reference to the $n$-th component, we repeat modifying the generators $\left\{y^{1}, y^{2}, \ldots, y^{r}\right\}$ so that an additional condition

$$
\left|y_{n}^{i}-z_{n}\right|<1 \quad(i=1,2, \ldots, r)
$$

is satisfied. By applying the same procedure for all other components, we arrive at a representation of $z$ as a convex combination of points in $S \cap N(z)$. 
The modification procedure with reference to the $n$-th component is now described. Without loss of generality we may assume

$$
\left|y_{n}^{1}-z_{n}\right| \geq\left|y_{n}^{2}-z_{n}\right| \geq \cdots \geq\left|y_{n}^{r}-z_{n}\right| .
$$

Suppose that $\left|y_{n}^{1}-z_{n}\right| \geq 1$; otherwise we are done. We may assume $y_{n}^{1}-z_{n} \geq 1$, since the other case $y_{n}^{1}-z_{n} \leq-1$ can be treated in a similar manner.

We claim that there exists $\left\{y^{j_{1}}, \ldots, y^{j_{k}}\right\} \subseteq\left\{y^{2}, y^{3}, \ldots, y^{r}\right\}$ such that, for $i=1, \ldots, k$, the $n$-th component of $y^{j_{i}}$ is less than that of $z$ (i.e., $y_{n}^{j_{i}}<z_{n}$ ) and

$$
\sum_{i=1}^{k-1} \lambda_{j_{i}}<\lambda_{1} \leq \sum_{i=1}^{k} \lambda_{j_{i}} .
$$

This claim can be proved as follows. Let $I=\left\{i \mid y_{n}^{i}<z_{n}\right\}$. By $(8.1),(8.3)$, and $y_{n}^{1}-z_{n} \geq 1$ we obtain

$$
\begin{aligned}
0 & =\sum_{i=1}^{r} \lambda_{i}\left(y_{n}^{i}-z_{n}\right)=\sum_{i: y_{n}^{i}>z_{n}} \lambda_{i}\left(y_{n}^{i}-z_{n}\right)+\sum_{i: y_{n}^{i}<z_{n}} \lambda_{i}\left(y_{n}^{i}-z_{n}\right) \\
& \geq \lambda_{1}\left(y_{n}^{1}-z_{n}\right)+\sum_{i \in I} \lambda_{i}\left(y_{n}^{i}-z_{n}\right)=\lambda_{1}\left|y_{n}^{1}-z_{n}\right|-\sum_{i \in I} \lambda_{i}\left|y_{n}^{i}-z_{n}\right| \\
& \geq \lambda_{1}\left|y_{n}^{1}-z_{n}\right|-\sum_{i \in I} \lambda_{i}\left|y_{n}^{1}-z_{n}\right|=\left|y_{n}^{1}-z_{n}\right|\left(\lambda_{1}-\sum_{i \in I} \lambda_{i}\right)
\end{aligned}
$$

which shows $\lambda_{1} \leq \sum_{i \in I} \lambda_{i}$. Then there exists $k$ that satisfies 8.4).

For $i=1, \ldots, k$ we have $\left\|y^{1}-y^{j_{i}}\right\|_{\infty} \geq 2$, since $y_{n}^{1} \geq z_{n}+1$ and $y_{n}^{j_{i}}<z_{n}$. Define

$$
y^{+j_{i}}=\left\lceil\frac{y^{1}+y^{j_{i}}}{2}\right\rceil, \quad y^{-j_{i}}=\left\lfloor\frac{y^{1}+y^{j_{i}}}{2}\right\rfloor
$$

for $i=1, \ldots, k$. We have $y^{+j_{i}}, y^{-j_{i}} \in S$ for $i=1, \ldots, k$ by the definition 2.4 of a discrete midpoint convex set. Among the terms in the convex combination 8.1) we consider the terms for $y^{1}, y^{j_{1}}, \ldots, y^{j_{k}}$. Since $y^{1}+y^{j_{i}}=y^{+j_{i}}+y^{-j_{i}}$ for $i=1, \ldots, k-1$ and $y^{1}=y^{+j_{k}}+y^{-j_{k}}-y^{j_{k}}$, we have

$$
\begin{aligned}
& \lambda_{1} y^{1}+\sum_{i=1}^{k} \lambda_{j_{i}} y^{j_{i}} \\
& =\sum_{i=1}^{k-1} \lambda_{j_{i}}\left(y^{1}+y^{j_{i}}\right)+\left(\lambda_{1}-\sum_{i=1}^{k-1} \lambda_{j_{i}}\right) y^{1}+\lambda_{j_{k}} y^{j_{k}} \\
& =\sum_{i=1}^{k-1} \lambda_{j_{i}}\left(y^{+j_{i}}+y^{-j_{i}}\right)+\left(\lambda_{1}-\sum_{i=1}^{k-1} \lambda_{j_{i}}\right)\left(y^{+j_{k}}+y^{-j_{k}}\right)+\left(\sum_{i=1}^{k} \lambda_{j_{i}}-\lambda_{1}\right) y^{j_{k}} .
\end{aligned}
$$

By (8.4) all the coefficients in the last expression are nonnegative and their sum is equal to the sum of the coefficients of the first expression, which is $\lambda_{1}+\sum_{i=1}^{k} \lambda_{j_{i}}$.

We change the generators $\left\{y^{1}, y^{2}, \ldots, y^{r}\right\}$ in the representation 8.1 by deleting $y^{1}$ and $\left\{y^{j_{1}}, \ldots, y^{j_{k-1}}\right\}$ (and also $y^{j_{k}}$ if $\sum_{i=1}^{k} \lambda_{j_{i}}-\lambda_{1}=0$ ) and by adding $\left\{y^{+j_{1}}, \ldots, y^{+j_{k}}\right\}$ and $\left\{y^{-j_{1}}, \ldots, y^{-j_{k}}\right\}$. Since $\left|y_{n}^{+j_{i}}-z_{n}\right|<\left|y_{n}^{1}-z_{n}\right|$ and $\left|y_{n}^{-j_{i}}-z_{n}\right|<\left|y_{n}^{1}-z_{n}\right|$ for $i=1, \ldots, k$, the resulting set of generators is "lexicographically smaller" with respect to the discrepancy in the $n$-th components arranged as in 8.3 . By finiteness, the above procedure eventually terminates with $\left|y_{n}^{1}-z_{n}\right|<1$, which implies (8.2). 
We next apply the above procedure with reference to the $(n-1)$-st component. What is crucial here is that the condition $(8.2)$ is maintained in the modification of the generators. Indeed, for each $i$, the inequalities $\left|y_{n}^{+J_{i}}-z_{n}\right|<1$ and $\left|y_{n}^{-j_{i}}-z_{n}\right|<1$ follow from 8.2) and 8.5). Therefore, we can obtain a representation of the form of $(8.1)$ with

$$
\left|y_{n}^{i}-z_{n}\right|<1, \quad\left|y_{n-1}^{i}-z_{n-1}\right|<1 \quad(i=1,2, \ldots, r) .
$$

By continuing in this way for other components, we finally obtain a representation of the form of $(8.1)$ with $\left|y_{j}^{i}-z_{j}\right|<1$ for $j=1,2, \ldots, n$ and $i=1,2, \ldots, r$. This completes the proof of Proposition 2.5 (2).

\subsection{Proof of Theorem 2.7}

Let $S \subseteq \mathbb{Z}^{n}$ be a discrete midpoint convex set and $x, y \in S$, and consider the decomposition $y-x=\sum_{k=1}^{m}\left(\mathbf{1}_{A_{k}}-\mathbf{1}_{B_{k}}\right)$ in $(2.5)$. For the proof of Theorem 2.7 it suffices to prove that

$$
x+\sum_{k \in J}\left(\mathbf{1}_{A_{k}}-\mathbf{1}_{B_{k}}\right) \in S
$$

for any subset $J$ of $\{1,2, \ldots, m\}$. Indeed, Theorem 2.7 follows from 8.7 for $J$ and $\{1,2, \ldots, m\} \backslash$ $J$.

Our proof strategy is to relate the decomposition (2.5) to discrete midpoint convexity by showing an alternative construction of the decomposition (2.5) using operations of roundingup $\lceil x\rceil$ and rounding-down $\lfloor x\rfloor$. For any $x \in \mathbb{Z}^{n}$ we denote the positive support of $x$ by $\operatorname{supp}^{+}(x)=\left\{i \mid x_{i}>0\right\}$ and the negative support of $x$ by $\operatorname{supp}^{-}(x)=\left\{i \mid x_{i}<0\right\}$. We are concerned with a decomposition of an integer vector $x$ into a family $D(x)$ of vectors such that:

(C1) $d \in\{-1,0,+1\}^{n} \backslash\{\mathbf{0}\} \quad(\forall d \in D(x))$.

(C2) $\sum\{d \mid d \in D(x)\}=x$.

(C3) $\operatorname{supp}^{+}(d) \subseteq \operatorname{supp}^{+}(x), \quad \operatorname{supp}^{-}(d) \subseteq \operatorname{supp}^{-}(x) \quad(\forall d \in D(x))$.

(C4) $\left\{\operatorname{supp}^{+}(d) \mid d \in D(x)\right\}$ and $\left\{\operatorname{supp}^{-}(d) \mid d \in D(x)\right\}$ each form a chain (nested family) with respect to set inclusion.

(C5) $|D(x)|=\|x\|_{\infty}$.

(C6) The vectors in $D(x)$ form a chain (linearly ordered set) with respect to vector ordering.

We first introduce a recursive scheme for a decomposition of an integer vector $x$ into a family $D_{0}(x)$ of vectors, which satisfies (C1) to (C4) above. The family $D_{0}(x)$ is modified to $D_{1}(x)$ to meet (C5), and then to $D_{2}(x)$, which satisfies (C1) to (C6). This decomposition scheme, when applied to $y-x$ for $x, y \in \mathbb{Z}^{n}$, yields the decomposition (2.5), that is,

$$
D_{2}(y-x)=\left\{\mathbf{1}_{A_{k}}-\mathbf{1}_{B_{k}} \mid k=1, \ldots, m\right\} .
$$

Finally we show that, if $x, y \in S$, where $S$ is a discrete midpoint convex set, then the property (8.7) follows from the construction of $D_{2}(y-x)$. Having explained our proof strategy, we now begin the technical arguments. The proof consists of five steps. 
Step 1: For any $x \in \mathbb{Z}^{n}$, we define a family (multiset) $D_{0}(x)$ of vectors by the following recursive formula:

$$
D_{0}(x)= \begin{cases}\emptyset & (x=\mathbf{0}), \\ \{x\} & \left(\|x\|_{\infty}=1\right), \\ \{\lceil x / 2\rceil,\lfloor x / 2\rfloor\} & \left(\|x\|_{\infty}=2\right), \\ D_{0}(\lceil x / 2\rceil) \cup D_{0}(\lfloor x / 2\rfloor) & \left(\|x\|_{\infty} \geq 3\right) .\end{cases}
$$

Lemma 8.1. For any $x \in \mathbb{Z}^{n}, D_{0}(x)$ satisfies (C1), (C2), (C3), and (C4).

Proof. (C1) and (C3) are obvious from the definition (8.9).

(C2) If $\|x\|_{\infty}=1$, we have $D_{0}(x)=\{x\}$ and the claim is obviously true. If $\|x\|_{\infty}=2$, we have $D_{0}(x)=\{\lceil x / 2\rceil,\lfloor x / 2\rfloor\}$, and the claim is true since $\lceil x / 2\rceil+\lfloor x / 2\rfloor=x$. We prove the claim for $\|x\|_{\infty} \geq 3$ by induction on $\|x\|_{\infty}$. If $\|x\|_{\infty} \geq 3$, we have $\|\lceil x / 2\rceil\|_{\infty}<\|x\|_{\infty}$ and $\|\lfloor x / 2\rfloor\|_{\infty}<\|x\|_{\infty}$. Hence, the induction hypothesis implies

$$
\sum\left\{d \mid d \in D_{0}(\lceil x / 2\rceil)\right\}=\lceil x / 2\rceil, \quad \sum\left\{d \mid d \in D_{0}(\lfloor x / 2\rfloor)\right\}=\lfloor x / 2\rfloor .
$$

Therefore, $\sum\left\{d \mid d \in D_{0}(x)\right\}=\sum\left\{d \mid d \in D_{0}(\lceil x / 2\rceil)\right\}+\sum\left\{d \mid d \in D_{0}(\lfloor x / 2\rfloor)\right\}=\lceil x / 2\rceil+$ $\lfloor x / 2\rfloor=x$.

(C4) The definition 8.9 implies:

$$
\begin{array}{ll}
x_{i} \geq x_{j} \Rightarrow d_{i} \geq d_{j} & \left(\forall i, j \in\{1,2, \ldots, n\}, \forall d \in D_{0}(x)\right), \\
x_{i}=x_{j} \Rightarrow d_{i}=d_{j} & \left(\forall i, j \in\{1,2, \ldots, n\}, \forall d \in D_{0}(x)\right) .
\end{array}
$$

Therefore, for any $d, d^{\prime} \in D_{0}(x)$, we have $\operatorname{supp}^{+}(d) \subseteq \operatorname{supp}^{+}\left(d^{\prime}\right)$ or $\operatorname{supp}^{+}(d) \supseteq \operatorname{supp}^{+}\left(d^{\prime}\right)$, which means that $\left\{\operatorname{supp}^{+}(d) \mid d \in D_{0}(x)\right\}$ forms a chain. Similarly for $\left\{\operatorname{supp}^{-}(d) \mid d \in\right.$ $\left.D_{0}(x)\right\}$.

Example 8.1. For $x=(5,3,-3,-5), D_{0}(x)$ is given as follows:

$$
\begin{aligned}
& D_{0}((5,3,-3,-5)) \\
&=D_{0}((3,2,-1,-2)) \cup D_{0}((2,1,-2,-3)) \\
&=D_{0}((2,1,0,-1)) \cup D_{0}((1,1,-1,-1)) \cup D_{0}((1,1,-1,-1)) \cup D_{0}((1,0,-1,-2)) \\
&=D_{0}((1,1,0,0)) \cup D_{0}((1,0,0,-1)) \cup D_{0}((1,1,-1,-1)) \cup D_{0}((1,1,-1,-1)) \\
& \cup D_{0}((1,0,0,-1)) \cup D_{0}((0,0,-1,-1)) \\
&=\{(1,1,0,0),(1,0,0,-1),(1,1,-1,-1),(1,1,-1,-1),(1,0,0,-1),(0,0,-1,-1)\} .
\end{aligned}
$$

The recursive process of the definition of $D_{0}(x)$ can be represented by the binary tree shown in Fig. 6. This tree has eleven vertices $\left\{v_{1}, \ldots, v_{11}\right\}$, and each vertex is associated with a vector that appears in the recursive definition of $D_{0}(x)$ above.

Concerning (C4) we have $\left\{\operatorname{supp}^{+}(d) \mid d \in D_{0}(x)\right\}=\{\{1,2\},\{1\}, \emptyset\}$ and $\left\{\operatorname{supp}^{-}(d) \mid d \in\right.$ $\left.D_{0}(x)\right\}=\{\{3,4\},\{4\}, \emptyset\}$, which are chains indeed. $D_{0}(x)$ does not satisfy (C5), since $\left|D_{0}(x)\right|=$ 6 and $\|x\|_{\infty}=5$. $D_{0}(x)$ does not satisfy (C6) either, since neither $(1,0,0,-1) \leq(1,1,-1,-1)$ nor $(1,0,0,-1) \geq(1,1,-1,-1)$ is true.

Just as in the above example, we can associate a binary tree with the recursive definition of $D_{0}(x)$. Let us denote this tree by $T_{0}(x)$ and the vector associated with a vertex $v$ of this tree by $\varphi(v)$. Then $D_{0}(x)$ is given as $D_{0}(x)=\left\{\varphi(v) \mid v\right.$ is a leaf of $\left.T_{0}(x)\right\}$. We assume that the left child of a vertex $v$ corresponds to $\lceil\varphi(v) / 2\rceil$ and the right child to $\lfloor\varphi(v) / 2\rfloor$; see Fig. 6. For a vertex $v$ in $T_{0}(x)$, the tree $T_{0}(\varphi(v))$ can be regarded as a subtree of $T_{0}(x)$. 


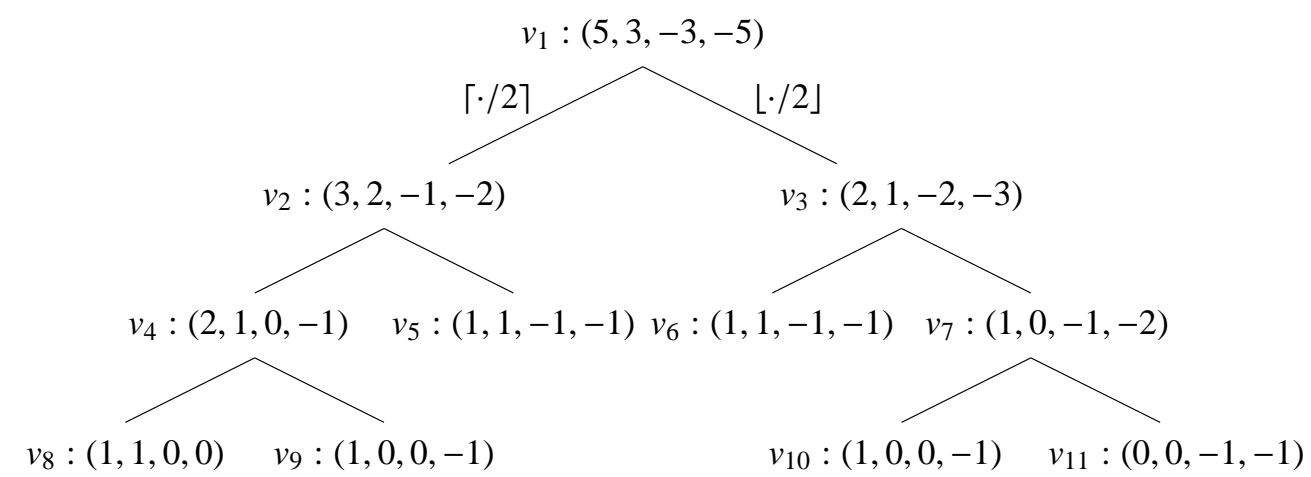

Figure 6: Tree of $D_{0}(x)$ for $x=(5,3,-3,-5)$ in Example 8.1

Step 2: We next modify the definition of $D_{0}(x)$ so as to meet (C5) in addition to (C1)-(C4). Note that $\left|D_{0}(x)\right| \geq\|x\|_{\infty}$ by (C1) and (C2). To state two lemmas we introduce notations

$$
\|x\|_{\infty}^{+}=\max \left(0, x_{1}, x_{2}, \ldots, x_{n}\right), \quad\|x\|_{\infty}^{-}=\max \left(0,-x_{1},-x_{2}, \ldots,-x_{n}\right) .
$$

We have $\|x\|_{\infty}=\max \left(\|x\|_{\infty}^{+},\|x\|_{\infty}^{-}\right)$. Let us say that an integer vector $x$ is critical if $\|x\|_{\infty} \geq 3$, $\|x\|_{\infty}$ is odd, and $\|x\|_{\infty}^{+}=\|x\|_{\infty}^{-}$. A vertex $v$ of the tree $T_{0}(x)$ is also called critical if the associated vector $\varphi(v)$ is critical. We modify the definition $(8.9)$ of $D_{0}(x)$ for critical $x$.

Lemma 8.2. For $x \in \mathbb{Z}^{n}$ with $\|x\|_{\infty} \geq 2$, we have

$$
\|\lceil x / 2\rceil\|_{\infty}+\|\lfloor x / 2\rfloor\|_{\infty}= \begin{cases}\|x\|_{\infty}+1 & (x: \text { critical }), \\ \|x\|_{\infty} & (x: \text { non-critical }) .\end{cases}
$$

Proof. Let $\ell=\|x\|_{\infty}$. If $x$ is critical, we have $\|\lceil x / 2\rceil\|_{\infty}=\|\lfloor x / 2\rfloor\|_{\infty}=(\ell+1) / 2$ and hence the claim holds. Suppose that $x$ is not critical. If $\ell$ is even, we have $\|\lceil x / 2\rceil\|_{\infty}=\|\lfloor x / 2\rfloor\|_{\infty}=\ell / 2$ and hence the claim holds. If $\ell$ is odd and $\|x\|_{\infty}^{+}>\|x\|_{\infty}^{-}$, we have $\|\lceil x / 2\rceil\|_{\infty}=(\ell+1) / 2$ and $\|\lfloor x / 2\rfloor\|_{\infty}=(\ell-1) / 2$, and hence the claim holds. If $\ell$ is odd and $\|x\|_{\infty}^{+}<\|x\|_{\infty}^{-}$, we have $\|\lceil x / 2\rceil\|_{\infty}=(\ell-1) / 2$ and $\|\lfloor x / 2\rfloor\|_{\infty}=(\ell+1) / 2$, and hence the claim holds.

Lemma 8.3. Let $x \in \mathbb{Z}^{n}$.

(1) If $\|x\|_{\infty}^{+}>\|x\|_{\infty}^{-}$and $\|x\|_{\infty} \geq 2$, then $y=\lceil x / 2\rceil$ satisfies $\|y\|_{\infty}^{+}>\|y\|_{\infty}^{-}$.

(2) If $\|x\|_{\infty}^{+}<\|x\|_{\infty}^{-}$and $\|x\|_{\infty} \geq 2$, then $z=\lfloor x / 2\rfloor$ satisfies $\|z\|_{\infty}^{+}<\|z\|_{\infty}^{-}$.

Proof. (1) If $\|x\|_{\infty}^{+}=2 k$ (even), then $\|y\|_{\infty}^{+}=k$ and $\|y\|_{\infty}^{-} \leq k-1$. If $\|x\|_{\infty}^{+}=2 k+1$ (odd), then $\|y\|_{\infty}^{+}=k+1$ and $\|y\|_{\infty}^{-} \leq k$. (2) This can be shown similarly.

Let $v$ be a critical vertex of $T_{0}(x)$ and $y=\varphi(v)$. Let $v^{\oplus}$ and $v^{\ominus}$, respectively, be the leftmost and rightmost leaves of the subtree below $v$. Define $d^{\oplus}(v)=\varphi\left(v^{\oplus}\right)$ and $d^{\ominus}(v)=\varphi\left(v^{\ominus}\right)$. For each vertex $w$ on the path between the left child of $v$ and the leaf $v^{\oplus}$ (inclusive), we have $\|\varphi(w)\|_{\infty}^{+}>\|\varphi(w)\|_{\infty}^{-}$by Lemma $8.3(1)$. In particular, $\left\|d^{\oplus}(v)\right\|_{\infty}^{+}>\left\|d^{\oplus}(v)\right\|_{\infty}^{-}$, which implies $d^{\oplus}(v) \in\{0,+1\}^{n}$ since $d^{\oplus}(v) \in\{-1,0,+1\}^{n}$. Similarly, we have $d^{\ominus}(v) \in\{0,-1\}^{n}$ by Lemma $8.3(2)$.

Example 8.2. The vector $x=(5,3,-3,-5)$ in Example 8.1 is critical, since $\|x\|_{\infty}=5 \geq 3$ is odd and $\|x\|_{\infty}^{+}=\|x\|_{\infty}^{-}=5$. Accordingly, in Fig. 6, the root vertex $v_{1}$ is critical. We have $v_{1}^{\oplus}=v_{8}, v_{1}^{\ominus}=v_{11}, d^{\oplus}\left(v_{1}\right)=(1,1,0,0)$, and $d^{\ominus}\left(v_{1}\right)=(0,0,-1,-1)$. 
Lemma 8.4. For distinct critical vertices $u$ and $v$ in $T_{0}(x)$, the leaves $u^{\oplus}, u^{\ominus}, v^{\oplus}$, and $v^{\ominus}$ are all distinct.

Proof. For a critical vertex $v$, there is no critical vertex on the path between the left child of $v$ and the leaf $v^{\oplus}$ by Lemma 8.3 (1), and there is no critical vertex on the path between the right child of $v$ and the leaf $v^{\ominus}$ by Lemma 8.3 (2). The claim follows from this fact.

With the above preparations we are now able to define $D_{1}(x)$ as follows 8

$$
\begin{aligned}
D_{1}(x)= & \left(D_{0}(x) \backslash\left\{d^{\oplus}(v), d^{\ominus}(v) \mid v \in T_{0}(x) \text { is critical }\right\}\right) \\
& \cup\left\{d^{\oplus}(v)+d^{\ominus}(v) \mid v \in T_{0}(x) \text { is critical }\right\} .
\end{aligned}
$$

Example 8.3. In Fig. 6 for $x=(5,3,-3,-5), v_{1}$ is the only critical vector with $d^{\oplus}\left(v_{1}\right)=$ $(1,1,0,0)$ and $d^{\ominus}\left(v_{1}\right)=(0,0,-1,-1) . D_{1}(x)$ is obtained from $D_{0}(x)$ in Example 8.1 by deleting $(1,1,0,0)$ and $(0,0,-1,-1)$, and adding their sum $(1,1,-1,-1)$. Hence $D_{1}((5,3,-3,-5))$ $=\{(1,0,0,-1),(1,1,-1,-1),(1,1,-1,-1),(1,0,0,-1),(1,1,-1,-1)\}$.

An alternative definition of $D_{1}(x)$, which modifies the recursive definition of $D_{0}(x)$, is also possible. For a critical vector $y$ appearing in the recursive definition of $D_{0}(x)$, we define $d^{\oplus}(y)=\varphi\left(v^{\oplus}\right)$ and $d^{\ominus}(y)=\varphi\left(v^{\ominus}\right)$ by choosing any vertex $v$ such that $y=\varphi(v)$. This is welldefined since, for two critical vertices $u$ and $v$ with $\varphi(u)=\varphi(v)$, we have $\varphi\left(u^{\oplus}\right)=\varphi\left(v^{\oplus}\right)$ and $\varphi\left(u^{\ominus}\right)=\varphi\left(v^{\ominus}\right)$. The recursive definition of $D_{1}(x)$ reads as follows:

$$
D_{1}(x)= \begin{cases}\emptyset & (x=\mathbf{0}) \\ \{x\} & \left(\|x\|_{\infty}=1\right) \\ \{\lceil x / 2\rceil,\lfloor x / 2\rfloor\} & \left(\|x\|_{\infty}=2\right) \\ D_{1}(\lceil x / 2\rceil) \cup D_{1}(\lfloor x / 2\rfloor) & \left(\|x\|_{\infty} \geq 3, x: \text { non-critical }\right) \\ \left(D_{1}(\lceil x / 2\rceil) \backslash\left\{d^{\oplus}(x)\right\}\right) \cup & \left(D_{1}(\lfloor x / 2\rfloor) \backslash\left\{d^{\ominus}(x)\right\}\right) \cup\left\{d^{\oplus}(x)+d^{\ominus}(x)\right\} \\ & \left(\|x\|_{\infty} \geq 3, x: \text { critical }\right) .\end{cases}
$$

In the last case, where $\|x\|_{\infty} \geq 3$ and $x$ is critical, the vectors $d^{\oplus}(x)$ and $d^{\ominus}(x)$ are deleted from $D_{1}(\lceil x / 2\rceil)$ and $D_{1}(\lfloor x / 2\rfloor)$, respectively, and their sum $d^{\oplus}(x)+d^{\ominus}(x) \in\{-1,0,+1\}^{n}$ is added instead.

Lemma 8.5. $D_{1}(x)$ satisfies $(\mathrm{C} 5)$ in addition to $(\mathrm{C} 1)-(\mathrm{C} 4)$.

Proof. First note that $d^{\oplus}(x)+d^{\ominus}(x) \in\{-1,0,+1\}^{n} \backslash\{\mathbf{0}\}$. Then it is easy to see that (C1)-(C4) for $D_{0}(x)$ imply $(\mathrm{C} 1)-(\mathrm{C} 4)$ for $D_{1}(x)$.

The condition (C5) can be shown as follows. If $\|x\|_{\infty} \leq 2$, (C5) is obviously true by the definition. Let $\ell=\|x\|_{\infty} \geq 3$. To prove (C5) by induction, assume that (C5) is satisfied by $D_{1}(z)$ for any $z \in \mathbb{Z}^{n}$ with $\|z\|_{\infty}<\|x\|_{\infty}$. Since $\|\lceil x / 2\rceil\|_{\infty}<\|x\|_{\infty}$ and $\|\lfloor x / 2\rfloor\|_{\infty}<\|x\|_{\infty}$ by $\|x\|_{\infty} \geq 3$, the induction hypothesis shows

$$
\left|D_{1}(\lceil x / 2\rceil)\right|=\|\lceil x / 2\rceil\|_{\infty}, \quad\left|D_{1}(\lfloor x / 2\rfloor)\right|=\|\lfloor x / 2\rfloor\|_{\infty} .
$$

If $x$ is not critical, we have $\|\lceil x / 2\rceil\|_{\infty}+\|\lfloor x / 2\rfloor\|_{\infty}=\|x\|_{\infty}$ by Lemma 8.2, and

$$
\left|D_{1}(x)\right|=\left|D_{1}(\lceil x / 2\rceil)\right|+\left|D_{1}(\lfloor x / 2\rfloor)\right|=\|\lceil x / 2\rceil\|_{\infty}+\|\lfloor x / 2\rfloor\|_{\infty}=\|x\|_{\infty} .
$$

\footnotetext{
${ }^{8}$ Recall that $D_{0}(x)$ is a multiset. The first term on the right-hand side of 8.10 means that we decrease the multiplicities of $d^{\oplus}(v)$ and $d^{\ominus}(v)$ for each critical vertex $v$.
} 
If $x$ is critical, we have $\|\lceil x / 2\rceil\|_{\infty}+\|\lfloor x / 2\rfloor\|_{\infty}=\|x\|_{\infty}+1$ by Lemma 8.2, and

$$
\begin{aligned}
\left|D_{1}(x)\right| & =\left(\left|D_{1}(\lceil x / 2\rceil)\right|-1\right)+\left(\left|D_{1}(\lfloor x / 2\rfloor)\right|-1\right)+1 \\
& =\|\lceil x / 2\rceil\|_{\infty}+\|\lfloor x / 2\rfloor\|_{\infty}-1=\|x\|_{\infty} .
\end{aligned}
$$

In either case, $D_{1}(x)$ satisfies (C5).

Lemma 8.6. We have $\left\|d^{\circ}+d^{\bullet}\right\|_{\infty}=2$ for any two vectors $d^{\circ}, d^{\bullet} \in D_{1}(x)$.

Proof. If $\left\|d^{\circ}+d^{\bullet}\right\|_{\infty} \leq 1, x$ would be represented as a sum of $\left|D_{1}(x)\right|-1=\|x\|_{\infty}-1$ vectors with $\ell_{\infty}$-norm $\leq 1$, which is a contradiction.

We note that Lemma 8.6 can be adapted to any collection of vectors satisfying (C1)-(C5).

Step 3: Finally we modify $D_{1}(x)$ so as to meet (C6) in addition to (C1)-(C5). Suppose that there are two vectors $d^{\circ}, d^{\bullet} \in D_{1}(x)$ that are not comparable with each other, i.e., $d^{\circ} \not \leq d^{\bullet}$ and $d^{\bullet} \not \leq d^{\circ}$. Letting

$$
d^{\uparrow}=\left\lceil\frac{d^{\circ}+d^{\bullet}}{2}\right\rceil, \quad d^{\downarrow}=\left\lfloor\frac{d^{\circ}+d^{\bullet}}{2}\right\rfloor,
$$

we modify $D_{1}(x)$ to

$$
D_{1}^{\prime}(x)=\left(D_{1}(x) \backslash\left\{d^{\circ}, d^{\bullet}\right\}\right) \cup\left\{d^{\uparrow}, d^{\downarrow}\right\},
$$

where the incomparable pair $\left\{d^{\circ}, d^{\bullet}\right\}$ is replaced by a comparable pair $\left\{d^{\uparrow}, d^{\downarrow}\right\}$ with $d^{\uparrow} \geq d^{\downarrow}$.

Lemma 8.7. $D_{1}^{\prime}(x)$ satisfies $(\mathrm{C} 1)-(\mathrm{C} 5)$.

Proof. (C2) to (C5) for $D_{1}^{\prime}(x)$ are easy to see. As for (C1), $d^{\uparrow}$ and $d^{\downarrow}$ are obviously $\{-1,0,+1\}$ vectors. They are nonzero since $\left\|d^{\circ}+d^{\bullet}\right\|_{\infty}=2$ by Lemma 8.6 adapted to $D_{1}^{\prime}(x)$.

Repeated application of the modification (8.13) generates a sequence $D_{1}^{\prime}(x), D_{1}^{\prime \prime}(x), \ldots$, which ends up with $D_{2}(x)=\left\{d^{1}, d^{2}, \ldots, d^{\ell}\right\}$ satisfying the chain condition $d^{1} \leq d^{2} \leq \cdots \leq d^{\ell}$, where $\ell=\|x\|_{\infty}$. The recursive application of Lemma 8.7 shows that $D_{1}^{\prime}(x), D_{1}^{\prime \prime}(x), \ldots, D_{2}(x)$ each satisfy $(\mathrm{C} 1)-(\mathrm{C} 5)$.

Example 8.4. In Example $8.3, D_{1}((5,3,-3,-5))$ contains an incomparable pair of vectors $d^{\circ}=(1,1,-1,-1)$ and $d^{\bullet}=(1,0,0,-1)$. We have $d^{\uparrow}=(1,1,0,-1)$ and $d^{\downarrow}=(1,0,-1,-1)$ in $(8.12)$, and $D_{2}((5,3,-3,-5))=\{(1,0,-1,-1),(1,0,-1,-1),(1,1,-1,-1),(1,1,0,-1)$, $(1,1,0,-1)\}$.

Step 4: For $x, y \in \mathbb{Z}^{n}$, consider $D_{2}(y-x)=\left\{d^{1}, d^{2}, \ldots, d^{m}\right\}$ with $d^{1} \leq d^{2} \leq \cdots \leq d^{m}$, where $m=\|y-x\|_{\infty}$. As is announced in (8.8), we have the following relation to the decomposition in (2.5).

Lemma 8.8. $\quad D_{2}(y-x)=\left\{\mathbf{1}_{A_{k}}-\mathbf{1}_{B_{k}} \mid k=1, \ldots, m\right\}$.

Proof. First recall that $y-x=\sum_{k=1}^{m}\left(\mathbf{1}_{A_{k}}-\mathbf{1}_{B_{k}}\right), A_{1} \subseteq A_{2} \subseteq \cdots \subseteq A_{m} \subseteq \operatorname{supp}^{+}(x-y)$, $B_{m} \subseteq B_{m-1} \subseteq \cdots \subseteq B_{1} \subseteq \operatorname{supp}^{-}(x-y)$, and $\mathbf{1}_{A_{1}}-\mathbf{1}_{B_{1}} \leq \mathbf{1}_{A_{2}}-\mathbf{1}_{B_{2}} \leq \cdots \leq \mathbf{1}_{A_{m}}-\mathbf{1}_{B_{m}}$ in the decomposition in 2.5 ). For each vector $d^{k}$ in $D_{2}(y-x)$, let $d_{+}^{k}, d_{-}^{k}$, and $d_{0}^{k}$ denote its restriction to (subvector on) to $\operatorname{supp}^{+}(y-x), \operatorname{supp}^{-}(y-x)$, and $\{1, \ldots, n\} \backslash\left(\operatorname{supp}^{+}(y-x) \cup \operatorname{supp}^{-}(y-x)\right)$, respectively; see Fig. 3 . The properties $(\mathrm{C} 1),(\mathrm{C} 3)$, and (C6) of $D_{2}(y-x)$ imply

$$
\mathbf{0} \leq d_{+}^{1} \leq d_{+}^{2} \leq \cdots \leq d_{+}^{m} \leq \mathbf{1}, \quad-\mathbf{1} \leq d_{-}^{1} \leq d_{-}^{2} \leq \cdots \leq d_{-}^{m} \leq \mathbf{0}, \quad d_{0}^{1}=d_{0}^{2}=\cdots=d_{0}^{m}=\mathbf{0} .
$$

Then we must have $d_{+}^{k}=\mathbf{1}_{A_{k}}$ and $d_{-}^{k}=-\mathbf{1}_{B_{k}}$ for $k=1, \ldots, m$ by the properties (C2) and (C5). 
Step 5: In Step 5, $S$ is assumed to be a discrete midpoint convex set in $\mathbb{Z}^{n}$ and $x, y \in S$. For any subset $E$ of $D_{i}(y-x)$ with $i=0,1$, or 2 , we consider the condition

$$
x+\sum\{d \mid d \in E\} \in S .
$$

Note that (8.14) holds for $E=\emptyset$ by $x \in S$, and for $E=D_{i}(y-x)$ by (C2) and $y \in S$. It should be clear that 8.14 corresponds to 8.7 .

\section{Lemma 8.9.}

(1) (8.14) holds for any $E \subseteq D_{0}(y-x)$.

(2) (8.14) holds for any $E \subseteq D_{1}(y-x)$.

Proof. (1) This is trivially true if $\|y-x\|_{\infty} \leq 1$. If $\|y-x\|_{\infty}=2$, we have $D_{0}(y-x)=$ $\{\lceil(y-x) / 2\rceil,\lfloor(y-x) / 2\rfloor\}$. Since $S$ is a discrete midpoint convex set, we have

$$
\begin{aligned}
& x+\lceil(y-x) / 2\rceil=\lceil(x+y) / 2\rceil \in S, \\
& x+\lfloor(y-x) / 2\rfloor=\lfloor(x+y) / 2\rfloor \in S,
\end{aligned}
$$

which show 8.14$)$ for $E$ with $\emptyset \neq E \neq D_{0}(y-x)$.

Let $\|y-x\|_{\infty} \geq 3$. To prove the claim by induction, assume that 8.14 is true for all $x^{\prime}, y^{\prime} \in S$ with $\left\|y^{\prime}-x^{\prime}\right\|_{\infty}<\|y-x\|_{\infty}$ and for all $E \subseteq D_{0}\left(y^{\prime}-x^{\prime}\right)$. We now fix $E \subseteq D_{0}(y-x)$, and define $x^{\prime \prime}=\lfloor(x+y) / 2\rfloor$ and $y^{\prime \prime}=\lceil(x+y) / 2\rceil$, where $x^{\prime \prime}, y^{\prime \prime} \in S$ by (2.3). Since

$$
\begin{aligned}
\left\|y^{\prime \prime}-x\right\|_{\infty} & =\|\lceil(y-x) / 2\rceil\|_{\infty}<\|y-x\|_{\infty}, \\
\left\|y-x^{\prime \prime}\right\|_{\infty} & =\|\lceil(y-x) / 2\rceil\|_{\infty}<\|y-x\|_{\infty}
\end{aligned}
$$

by $\|y-x\|_{\infty} \geq 3$, the induction hypothesis for $\left(x, y^{\prime \prime}\right)$ and $\left(x^{\prime \prime}, y\right)$ shows

$$
\begin{aligned}
& u=x+\sum\left\{d \mid d \in E \cap D_{0}(\lceil(y-x) / 2\rceil)\right\} \in S, \\
& v=x^{\prime \prime}+\sum\left\{d \mid d \in E \cap D_{0}(\lceil(y-x) / 2\rceil)\right\} \in S,
\end{aligned}
$$

where $D_{0}\left(y^{\prime \prime}-x\right)=D_{0}\left(y-x^{\prime \prime}\right)=D_{0}(\lceil(y-x) / 2\rceil)$ is used. Since

$$
\left.\|v-u\|_{\infty}=\left\|x^{\prime \prime}-x\right\|_{\infty}=\| \mathrm{L}(y-x) / 2\right\rfloor\left\|_{\infty}<\right\| y-x \|_{\infty},
$$

we can also use the induction hypothesis for $(u, v)$ to obtain

$$
w=u+\sum\left\{d \mid d \in E \backslash D_{0}(\lceil(y-x) / 2\rceil)\right\} \in S,
$$

where $D_{0}(v-u)=D_{0}(\lfloor(y-x) / 2\rfloor) \supseteq E \backslash D_{0}(\lceil(y-x) / 2\rceil)$ by $D_{0}(\lceil(y-x) / 2\rceil) \cup D_{0}(\lfloor(y-x) / 2\rfloor)=$ $D_{0}(y-x) \supseteq E$. Substituting 8.15 into 8.17$)$ we obtain

$$
w=x+\sum\{d \mid d \in E\} \in S,
$$

which is nothing but (8.14).

(2) This follows from (1), since each vector of $D_{1}(y-x)$ is a sum of some vectors of $D_{0}(y-x)$.

Recall from Step 3 that $D_{2}(y-x)$ is constructed from $D_{1}(y-x)$ by repeated modification in 8.13 , which changes an incomparable pair $\left\{d^{\circ}, d^{\bullet}\right\}$ to a comparable pair $\left\{d^{\uparrow}, d^{\downarrow}\right\}$ defined by $(8.12)$. 
Lemma 8.10. Let $D_{1}^{\prime}$ be obtained from $D_{1}(y-x)$ as in (8.13). Then (8.14) holds for any $E \subseteq D_{1}^{\prime}$.

Proof. In accordance with 8.13$)$, we have $D_{1}^{\prime}=\left(D_{1}(y-x) \backslash\left\{d^{\circ}, d^{\bullet}\right\}\right) \cup\left\{d^{\uparrow}, d^{\downarrow}\right\}$. We have four cases to consider.

1. If $d^{\uparrow} \notin E$ and $d^{\downarrow} \notin E$, we have $E \subseteq D_{1}(y-x)$, and therefore (8.14) holds by Lemma 8.9 (2).

2. If $d^{\uparrow} \in E$ and $d^{\downarrow} \in E$, we have $d^{\uparrow}+d^{\downarrow}=d^{\circ}+d^{\bullet}$ and $\left(E \backslash\left\{d^{\uparrow}, d^{\downarrow}\right\}\right) \cup\left\{d^{\circ}, d^{\bullet}\right\} \subseteq D_{1}(y-x)$. Therefore,

$$
x+\sum\{d \mid d \in E\}=x+\sum\left\{d \mid d \in E \backslash\left\{d^{\uparrow}, d^{\downarrow}\right\}\right\}+d^{\circ}+d^{\bullet} \in S
$$

by Lemma 8.9 (2).

3. If $d^{\uparrow} \in E$ and $d^{\downarrow} \notin E$, both points

$$
\begin{aligned}
& y=x+\sum\left\{d \mid d \in E \backslash\left\{d^{\uparrow}\right\}\right\}+d^{\circ}+d^{\bullet}, \\
& z=x+\sum\left\{d \mid d \in E \backslash\left\{d^{\uparrow}\right\}\right\}
\end{aligned}
$$

belong to $S$ by Lemma 8.9 (2). We have $\lceil(y+z) / 2\rceil \in S$ since $\|y-z\|_{\infty}=\left\|d^{\circ}+d^{\bullet}\right\|_{\infty}=2$ (cf. Lemma 8.6) and $S$ is a discrete midpoint convex set. Therefore,

$$
\lceil(y+z) / 2\rceil=x+\sum\left\{d \mid d \in E \backslash\left\{d^{\uparrow}\right\}\right\}+d^{\uparrow} \in S,
$$

which shows (8.14).

4. If $d^{\uparrow} \notin E$ and $d^{\downarrow} \in E$, we can show 8.14 in a similar manner.

Lemma 8.11. 8.14) holds for any $E \subseteq D_{2}(y-x)$.

Proof. Repeated application of the modification $(8.13)$ generates a sequence $D_{1}^{\prime}, D_{1}^{\prime \prime}, \ldots$ that ends with $D_{2}(x-y)$. The recursive application of Lemma 8.10 shows that 8.14 ) holds for any $E \subseteq D_{1}^{\prime}, E \subseteq D_{1}^{\prime \prime}, \ldots, E \subseteq D_{2}(y-x)$.

Lemma 8.11 with Lemma 8.8 establishes Theorem 2.7

\section{Acknowledgements}

The authors thank Hiroshi Hirai for discussion about L-extendable functions and Kazuya Tsurumi for Remark 3.1. This research was initiated at the Trimester Program "Combinatorial Optimization" at Hausdorff Institute of Mathematics, 2015. This work was supported by The Mitsubishi Foundation, CREST, JST, Grant Number JPMJCR14D2, Japan, and JSPS KAKENHI Grant Numbers JP26350430, JP26280004, JP24300003, JP16K00023, JP17K00037. 


\section{A Equivalence of Integral Convexity and Global Weak DMC}

We prove that the global weak discrete midpoint convexity of a function implies the integral convexity even without assuming the integral convexity of the effective domain.

Theorem A.1. For a function $f: \mathbb{Z}^{n} \rightarrow \mathbb{R} \cup\{+\infty\}$, the following properties are equivalent:

(a) $f$ is integrally convex.

(b) For every $x, y \in \operatorname{dom} f$ with $\|x-y\|_{\infty} \geq 2$ we have

$$
f(x)+f(y) \geq 2 \tilde{f}\left(\frac{x+y}{2}\right) .
$$

Proof. Obviously, (a) implies (b). To prove "(b) $\Rightarrow$ (a)" by contradiction, assume that there exist $x \in \overline{\operatorname{dom} f}$ and $y^{1}, \ldots, y^{m} \in \operatorname{dom} f$ such that

$$
x=\sum_{i=1}^{m} \lambda_{i} y^{i}, \quad \tilde{f}(x)>\sum_{i=1}^{m} \lambda_{i} f\left(y^{i}\right),
$$

where $\sum_{i=1}^{m} \lambda_{i}=1$ and $\lambda_{i}>0(i=1, \ldots, m)$. For each $j=n, n-1, \ldots, 1$, we look at the $j$-th component of the generating points $y^{i}$.

Let $j=n$ and define

$$
\alpha_{n}=\min _{i} y_{n}^{i}, \quad \beta_{n}=\max _{i} y_{n}^{i}, \quad I_{\min }=\left\{i \mid y_{n}^{i}=\alpha_{n}\right\}, \quad I_{\max }=\left\{i \mid y_{n}^{i}=\beta_{n}\right\} .
$$

If $\beta_{n}-\alpha_{n} \leq 1$, we are done with $j=n$. Suppose that $\beta_{n}-\alpha_{n} \geq 2$. By translation and reversal of the $n$-th coordinate, we may assume $0 \leq x_{n} \leq 1, \alpha_{n} \leq 0$, and $\beta_{n} \geq 2$. By renumbering the generators we may assume $1 \in I_{\min }$ and $2 \in I_{\max }$, i.e., $y_{n}^{1}=\alpha_{n}$ and $y_{n}^{2}=\beta_{n}$. We have $\left\|y^{1}-y^{2}\right\|_{\infty} \geq 2$.

By A.1 for $\left(y^{1}, y^{2}\right)$ and the definition of $\tilde{f}$ we have

$$
f\left(y^{1}\right)+f\left(y^{2}\right) \geq 2 \tilde{f}\left(\frac{y^{1}+y^{2}}{2}\right)=2 \sum_{k=1}^{l} \mu_{k} f\left(z^{k}\right),
$$

where

$$
\frac{y^{1}+y^{2}}{2}=\sum_{k=1}^{l} \mu_{k} z^{k}, \quad z^{k} \in N\left(\frac{y^{1}+y^{2}}{2}\right) \cap \operatorname{dom} f \quad(k=1, \ldots, l)
$$

with $\mu_{k}>0(k=1, \ldots, l)$ and $\sum_{k=1}^{l} \mu_{k}=1$. The inequality A.4 implies, with notation $\lambda=\min \left(\lambda_{1}, \lambda_{2}\right)$, that

$$
\lambda_{1} f\left(y^{1}\right)+\lambda_{2} f\left(y^{2}\right) \geq\left(\lambda_{1}-\lambda\right) f\left(y^{1}\right)+\left(\lambda_{2}-\lambda\right) f\left(y^{2}\right)+2 \lambda \sum_{k=1}^{l} \mu_{k} f\left(z^{k}\right) .
$$

Hence

$$
\begin{aligned}
\sum_{i=1}^{m} \lambda_{i} f\left(y^{i}\right) & =\left(\lambda_{1} f\left(y^{1}\right)+\lambda_{2} f\left(y^{2}\right)\right)+\sum_{i=3}^{m} \lambda_{i} f\left(y^{i}\right) \\
& \geq\left(\lambda_{1}-\lambda\right) f\left(y^{1}\right)+\left(\lambda_{2}-\lambda\right) f\left(y^{2}\right)+2 \lambda \sum_{k=1}^{l} \mu_{k} f\left(z^{k}\right)+\sum_{i=3}^{m} \lambda_{i} f\left(y^{i}\right) .
\end{aligned}
$$


Since

$$
x=\left(\lambda_{1}-\lambda\right) y^{1}+\left(\lambda_{2}-\lambda\right) y^{2}+2 \lambda \sum_{k=1}^{l} \mu_{k} z^{k}+\sum_{i=3}^{m} \lambda_{i} y^{i},
$$

we have obtained another representation of the form (A.2).

With reference to this new representation we define $\hat{\alpha}_{n}, \hat{\beta}_{n}, \hat{I}_{\min }$, and $\hat{I}_{\max }$, as in A.3. Since $\beta_{n}-\alpha_{n} \geq 2$, we have

$$
\alpha_{n}+1 \leq\left(y_{n}^{1}+y_{n}^{2}\right) / 2 \leq \beta_{n}-1,
$$

which implies $\alpha_{n}+1 \leq z_{n}^{k} \leq \beta_{n}-1$ for all $k$. Hence, $\alpha_{n} \leq \hat{\alpha}_{n}$ and $\hat{\beta}_{n} \leq \beta_{n}$. Moreover, if $\left(\hat{\alpha}_{n}, \hat{\beta}_{n}\right)=\left(\alpha_{n}, \beta_{n}\right)$, then $\left|\hat{I}_{\min }\right|+\left|\hat{I}_{\max }\right| \leq\left|I_{\min }\right|+\left|I_{\max }\right|-1$. Therefore, by repeating the above process with $j=n$, we eventually arrive at a representation of the form of (A.2) with $\beta_{n}-\alpha_{n} \leq 1$.

We next apply the above procedure for the $(n-1)$-st component. What is crucial here is that the condition $\beta_{n}-\alpha_{n} \leq 1$ is maintained in the modification of the generators by (A.4) for the $(n-1)$-st component. Indeed, for each $k$, the inequality $\alpha_{n} \leq z_{n}^{k} \leq \beta_{n}$ follows from $\alpha_{n} \leq\left(y_{n}^{1}+y_{n}^{2}\right) / 2 \leq \beta_{n}$ and $z^{k} \in N\left(\left(y^{1}+y^{2}\right) / 2\right)$. Therefore, we can obtain a representation of the form of A.2 with $\beta_{n}-\alpha_{n} \leq 1$ and $\beta_{n-1}-\alpha_{n-1} \leq 1$, where $\alpha_{n-1}=\min _{i} y_{n-1}^{i}$ and $\beta_{n-1}=\max _{i} y_{n-1}^{i}$.

Then we continue the above process for $j=n-2, n-3, \ldots, 1$, to finally obtain a representation of the form of A.2 with $\left|y_{j}^{i}-y_{j}^{i^{\prime}}\right| \leq 1$ for all $i, i^{\prime}$ and $j=1,2, \ldots, n$. This contradicts the definition of $\tilde{f}$.

\section{References}

[1] Ahuja, R.K., Magnanti, T.L., Orlin, J.B.: Network Flows-Theory, Algorithms and Applications. Prentice-Hall, Englewood Cliffs (1993)

[2] Beckenbach, E. F.: Convex functions. Bulletin American Mathematical Society 54, 439-460 (1948).

[3] Begen, M., Queyranne, M.: Appointment scheduling with discrete random durations. Mathematics of Operations Research 36, 240-257 (2011)

[4] De Loera, J.A., Hemmecke, R., Köppe, M.: Algebraic and Geometric Ideas in the Theory of Discrete Optimization. SIAM, Philadelphia (2013)

[5] Favati, P., Tardella, F.: Convexity in nonlinear integer programming. Ricerca Operativa 53, 3-44 (1990)

[6] Fujishige, S.: Submodular Functions and Optimization, 2nd edn. Annals of Discrete Mathematics 58, Elsevier, Amsterdam (2005)

[7] Fujishige, S.: Bisubmodular polyhedra, simplicial divisions, and discrete convexity. Discrete Optimization 12, 115-120 (2014)

[8] Fujishige, S., Murota, K.: Notes on L-/M-convex functions and the separation theorems. Mathematical Programming 88, 129-146 (2000)

[9] Hemmecke, R., Köppe, M., Lee, J., Weismantel, R.: Nonlinear integer programming. In: Jünger, M., et al. (eds.) 50 Years of Integer Programming 1958-2008: From The Early Years and State-of-the-Art, Chapter 15, pp. 561-618, Springer, Berlin (2010) 
[10] Hirai, H.: L-extendable functions and a proximity scaling algorithm for minimum cost multiflow problem. Discrete Optimization 18, 1-37 (2015)

[11] Hirai, H.: L-convexity on graph structures. Journal of the Operations Research Society of Japan 61, 71-109 (2018)

[12] Hirai, H., Iwamasa, Y.: On $k$-submodular relaxation. SIAM Journal on Discrete Mathematics 30, 1726-1736 (2016)

[13] Hochbaum, D.S.: Complexity and algorithms for nonlinear optimization problems. Annals of Operations Research 153, 257-296 (2007)

[14] Hochbaum, D.S., Shanthikumar, J.G.: Convex separable optimization is not much harder than linear optimization. Journal of the Association for Computing Machinery 37, 843-862 (1990)

[15] Ibaraki, T., Katoh, N.: Resource Allocation Problems: Algorithmic Approaches. MIT Press, Boston (1988)

[16] Iimura, T.: Discrete modeling of economic equilibrium problems. Pacific Journal of Optimization 6, 57-64 (2010)

[17] Iimura, T., Murota, K., Tamura, A.: Discrete fixed point theorem reconsidered. Journal of Mathematical Economics 41, 1030-1036 (2005)

[18] Iimura, T., Watanabe, T.: Existence of a pure strategy equilibrium in finite symmetric games where payoff functions are integrally concave. Discrete Applied Mathematics 166, 26-33 (2014)

[19] Iwata, S., Moriguchi, S., Murota, K.: A capacity scaling algorithm for M-convex submodular flow. Mathematical Programming 103, 181-202 (2005)

[20] Iwata, S., Shigeno, M.: Conjugate scaling algorithm for Fenchel-type duality in discrete convex optimization. SIAM Journal on Optimization 13, 204-211 (2002)

[21] Jensen, J. L. W. V.: Om konvekse Funktioner og Uligheder imellem Middelværdier. Mathematica Scandinavica 16B, 49-68 (1905). Also: Sur les fonctions convexes et les inégalités entre les valeurs moyennes. Acta Mathematica 30, 175-193 (1906)

[22] Katoh, N., Shioura, A., Ibaraki, T.: Resource allocation problems. In: Pardalos, P.M., Du, D.-Z., Graham, R.L. (eds.) Handbook of Combinatorial Optimization, 2nd ed., Vol. 5, pp. 2897-2988, Springer, Berlin (2013)

[23] Kolmogorov, V., Shioura, A.: New algorithms for convex cost tension problem with application to computer vision. Discrete Optimization 6, 378-393 (2009)

[24] Lee, J., Leyffer, S. (eds): Mixed Integer Nonlinear Programming, The IMA Volumes in Mathematics and its Applications 154, Springer, Berlin (2012)

[25] Lehmann, B., Lehmann, D., Nisan, N.: Combinatorial auctions with decreasing marginal utilities. Games and Economic Behavior 55, 270-296 (2006)

[26] Moriguchi, S., Murota, K., Tamura, A., Tardella, F.: Scaling and proximity properties of integrally convex functions. In: Seok-Hee Hong (ed.) ISAAC2016, Leibniz International Proceedings in Informatics (LIPIcs), 64, Article No. 57, pp.57:1-57:12 (2016) 
[27] Moriguchi, S., Murota, K., Tamura, A., Tardella, F.: Scaling, proximity, and optimization of integrally convex functions. Mathematical Programming. DOI: 10.1007/s10107018-1234-z (2018)

[28] Moriguchi, S., Tsuchimura, N.: Discrete L-convex function minimization based on continuous relaxation. Pacific Journal of Optimization 5, 227-236 (2009)

[29] Murota, K.: Discrete convex analysis. Mathematical Programming 83, 313-371 (1998)

[30] Murota, K.: Discrete Convex Analysis. SIAM, Philadelphia (2003)

[31] Murota, K.: Recent developments in discrete convex analysis. In: Cook, W., Lovász, L., Vygen, J. (eds.) Research Trends in Combinatorial Optimization, pp. 219-260. Springer, Berlin (2009)

[32] Murota, K.: Discrete convex analysis: A tool for economics and game theory. Journal of Mechanism and Institution Design 1, 151-273 (2016)

[33] Murota, K., Shioura, A.: Exact bounds for steepest descent algorithms of L-convex function minimization. Operations Research Letters 42, 361-366 (2014)

[34] Murota, K., Shioura, A., Yang, Z.: Time bounds for iterative auctions: A unified approach by discrete convex analysis. Discrete Optimization 19, 36-62 (2016)

[35] Murota, K., Tamura, A.: Proximity theorems of discrete convex functions. RIMS Preprint 1358, Kyoto University (2002)

[36] Onn, S.: Nonlinear Discrete Optimization: An Algorithmic Theory. European Mathematical Society, Zurich (2010)

[37] Shioura, A.: Algorithms for L-convex function minimization: Connection between discrete convex analysis and other research areas. Journal of the Operations Research Society of Japan 60, 216-243 (2017)

[38] Simchi-Levi, D., Chen, X., Bramel, J.: The Logic of Logistics: Theory, Algorithms, and Applications for Logistics Management, 3rd ed. Springer, New York (2014)

[39] Thomson, B.: Symmetric Properties of Real Functions. Marcel Dekker, New York (1994)

[40] Yang, Z.: Discrete fixed point analysis and its applications. Journal of Fixed Point Theory and Applications 6, 351-371 (2009)

[41] Zipkin, P.: On the structure of lost-sales inventory models. Operations Research 56, 937-944 (2008) 